JOURNAL OF THE

AMERICAN MATHEMATICAL SOCIETY

Volume 8, Number 4, October 1995

\title{
ON RANKS OF TWISTS OF ELLIPTIC CURVES AND POWER-FREE VALUES OF BINARY FORMS
}

\author{
C. L. STEWART AND J. TOP
}

Dedicated to Professor Wolfgang Schmidt on the occasion of his sixtieth birthday

\section{INTRODUCTION}

Let $E$ be an elliptic curve over $\mathbb{Q}$. By the rank of $E$ we shall mean the rank of the group of rational points of $E$. Mestre [31], improving on the work of Néron [34] (cf. [13], [39] and [46]), has shown that there is an infinite family of elliptic curves over $\mathbb{Q}$ with rank at least 12 . However, computational work (see [3], [4], [7] and [47]) suggests that a typical elliptic curve will have much smaller rank, with curves of rank 0 or 1 being predominant. Indeed, Brumer [6] has proved, subject to the Birch, Swinnerton-Dyer conjecture, the Shimura, Taniyama, Weil conjecture and the generalized Riemann hypothesis, that the average rank of an elliptic curve, ordered according to its Faltings height, is at most 2.3.

In this article we shall study the behaviour of the rank as we run over twists of a given elliptic curve over $\mathbb{Q}$. That is, we shall restrict our attention to families of elliptic curves defined over $\mathbb{Q}$ which are isomorphic over $\mathbb{C}$. There are families of quadratic, cubic, quartic and sextic twists (see, for example, Proposition 5.4 of Chapter X of [42]). Let $E$ be an elliptic curve over $\mathbb{Q}$ with Weierstrass equation $y^{2}=x^{3}+a x+b$ and for any non-zero integer $d$ let $E_{d}$ denote a quadratic twist of $E$ given by the equation $d y^{2}=x^{3}+a x+b$. Let $r(d)$ denote the rank of $E_{d}$. Note that if $d_{1}$ and $d_{2}$ are non-zero integers, then $E_{d_{1}}$ is isomorphic to $E_{d_{2}}$ over $\mathbb{Q}$ if and only if $d_{1} / d_{2}$ is the square of a rational number. Subject to the conjectures of Birch and Swinnerton-Dyer and of Shimura, Taniyama, and Weil, Goldfeld [14] conjectured in 1979 that

$$
\sum_{0<|d| \leq x} r(d) \sim \frac{1}{2} \sum_{0<|d| \leq x} 1 .
$$

Further, in 1960 Honda [18] (see also [38], p. 162) conjectured that the rank of any twist of a given elliptic curve $E$ over a number field $K$ is bounded by a constant which depends on $E$ and $K$ only. Some related work on ranks of twists may be found in [12], [22] and [35].

Received by the editors July 30, 1992 and, in revised form, August 10, 1994.

1991 Mathematics Subject Classification. Primary 11G05, 11 N36.

The first author's research was supported in part by a Killam Research Fellowship and by Grant A3528 from the Natural Sciences and Engineering Research Council of Canada.

(c)1995 American Mathematical Society 
In 1987, Zagier and Kramarz [47] examined cubic twists of the curve $E$ given by the affine equation $x^{3}+y^{3}=1$. In particular they looked at the twists $E_{d}$ of $E$ given by the equation $x^{3}+y^{3}=d$. They calculated the value of the $L$-series of $E_{d}$ and its derivative at 1 for all cube-free positive integers $d$ less than 70,000. Subject to the Birch and Swinnerton-Dyer conjecture their calculations suggest that a positive proportion of the twists $E_{d}$ of $E$ have rank even and at least 2 and a positive proportion have rank odd and at least 3 . The first theoretical results in this context are due to Gouvêa and Mazur [15]. Let $E$ be an elliptic curve over $\mathbb{Q}$, given by $y^{2}=x^{3}+a x+b$. For any nonzero integer $d$ let $E_{d}$ denote the curve given by $d y^{2}=x^{3}+a x+b$ and let $\varepsilon$ be a positive real number. For any real number $T$, let $S(T)$ denote the number of square-free integers $d$ with $|d| \leq T$ for which the rank of $E_{d}$ is even and at least 2 . They proved, subject to the parity conjecture (see $\S 12$ ), that there are positive numbers $C_{0}$ and $C_{1}$, which depend on $\varepsilon$ and $E$, such that whenever $T$ exceeds $C_{0}$ then $S(T)$ is at least $C_{1} T^{\frac{1}{2}-\varepsilon}$. Thus, in the terminology of Gouvêa and Mazur, $\frac{1}{2}$ is an exponent for $S(T)$, subject to the parity conjecture. In [28], L. Mai extended the work of Gouvêa and Mazur to the case of cubic twists of $x^{3}+y^{3}=1$. He proved, subject to the parity conjecture for cubic twists (see $\S 12$ ), that for each positive real number $\varepsilon$ there exist positive numbers $C_{2}$ and $C_{3}$, which depend on $\varepsilon$, such that for any real number $T$ larger than $C_{2}$ the number of cube-free integers $d$ with $|d| \leq T$ for which the rank of the curve given by $x^{3}+y^{3}=d$ is even and at least 2 is at least $C_{3} T^{\frac{2}{3}-\varepsilon}$.

Our aim in this paper is to generalize these results and to remove the dependence on the parity conjecture. Further we shall give a positive response to the question, posed by Gouvêa and Mazur [15], of whether there are positive exponents associated to functions counting the number of twists of an elliptic curve $E$ which have rank larger than 2 . For instance, we shall prove that there is a positive number $C_{4}$ such that if $T$ is larger than 657 , then the number of cube-free integers $d$ with $|d| \leq T$ for which the curve given by $x^{3}+y^{3}=d$ has rank at least 3 is at least $C_{4} T^{\frac{1}{6}}$. Furthermore, by employing a construction of Mestre [30], we shall prove that if $E$ is an elliptic curve over $\mathbb{Q}$ with $j$-invariant different from 0 and 1728 and, as usual, $E_{d}$ denotes the quadratic twist of $E$, then there are positive numbers $C_{5}$ and $C_{6}$ which depend on $E$, such that if $T$ exceeds $C_{5}$, then $S(T)$ is at least $C_{6} T^{\frac{1}{7}} /(\log T)^{2}$.

Our strategy will be to provide curves which are degree $2,3,4$ and 6 cyclic covers of the projective line and which have the property that their jacobian contains over $\mathbb{Q}$, up to isogeny, many copies of a given elliptic curve. This method is not new, indeed Tate and Shafarevich [45] used it in the context of finite fields instead of $\mathbb{Q}$.

For any positive integer $k$ and any integer $n$ we say that $n$ is $k$-free if $n$ is not divisible by the $k$ th power of a prime. To estimate the number of twists of large rank that our constructions yield we shall appeal to an estimate, of interest in its own right, for the number of $k$-free values below a given bound assumed by a binary form with integer coefficients evaluated at integer 
arguments. Our result depends upon recent work of Greaves [16] who studied the related problem of estimating the number of pairs of integers $(a, b)$ with $|a|$ and $|b|$ below a given bound for which $F(a, b)$ is $k$-free. To apply the work of Greaves we shall employ an argument due to Erdős and Mahler [11] and a result of Stewart [43] on the number of solutions of the Thue equation. Greaves' result sharpened work of Gouvêa and Mazur which in turn depended on a result of Hooley [19] which gives an asymptotic formula for the number of $k$-free positive integers up to $x$ represented by an irreducible polynomial with integer coefficients of degree $k+1 \quad(\geq 3)$ and having no fixed $k$ th power divisors.

Part of the research reported on here was done while the second author worked at Queen's University in Kingston, Ontario. During the remaining part he was employed by the Erasmus University in Rotterdam, the Netherlands. It is a pleasure to thank these institutions for their hospitality.

\section{Strategy}

Let $E$ be an elliptic curve defined over $\mathbb{Q}$. Our aim is to construct many twists of $E$ having large rank. To do so we shall regard $E$ as an elliptic curve over $\mathbb{Q}(t)$. We shall study twists $E_{D}$ of $E$ where $D$ is a polynomial in $\mathbb{Z}[t]$ of positive degree and where $E_{D}$ and $E$ are not isomorphic over $\mathbb{Q}(t)$ but become isomorphic over an extension $\mathbb{Q}(t, s)$ of $\mathbb{Q}(t)$ where $s^{n}=D(t)$ and $n$ is $2,3,4$ or 6 when we are considering quadratic, cubic, quartic or sextic twists respectively.

Our approach will be to first construct $D$ so that the rank of the group of $\mathbb{Q}(t)$-points of $E_{D}$ is large. Next we will appeal to a specialization argument to show that most specializations of $t$ to a rational number give an elliptic curve over $\mathbb{Q}$ with rank at least as large as the rank of $E_{D}$ over $\mathbb{Q}(t)$. Finally we shall use our result on $n$-free values assumed by binary forms to count the number of different twists we get by specializing. The binary form $F(X, Y)$ which we will arrive at is given by $F(X, Y)=Y^{l} D\left(\frac{X}{Y}\right)$ where $l$ is the smallest multiple of $n$ greater than or equal to the degree of $D$.

\section{Specialization}

Let $E$ be an elliptic curve defined over $\mathbb{Q}(t)$ which is not isomorphic over $\mathbb{Q}(t)$ to an elliptic curve defined over $\mathbb{Q}$. By specializing $t$ to a rational number $t_{0}$ one obtains in general an elliptic curve $E_{t_{0}} / \mathbb{Q}$ and a specialization homomorphism $\rho_{t_{0}}: E(\mathbb{Q}(t)) \rightarrow E_{t_{0}}(\mathbb{Q})$ from the group of $\mathbb{Q}(t)$-points of $E$ to the group of rational points of $E_{t_{0}}$. We shall make use of the following result due to Silverman.

Lemma 1. In the above situation,

$$
\rho_{t_{0}}: E(\mathbb{Q}(t)) \rightarrow E_{t_{0}}(\mathbb{Q})
$$

is an injective homomorphism for all but finitely many rational numbers $t_{0}$.

Proof. This is a special case of Theorem C of [40]; one takes the abelian variety to be an elliptic curve. 


\section{Calculation of the Rank of $E_{D}$ OVER $\mathbb{Q}(t)$}

Let $D(t)$ be a polynomial with integer coefficients and degree at least 1 and let $n$ be an integer larger than 1. Suppose that $D$ is not a perfect $p$ th power in $\mathbb{C}[t]$ for any prime $p$ which divides $n$. Let $C$ be a smooth, complete model of the curve given by $s^{n}=D(t)$ and let $H^{0}\left(C, \Omega_{C / Q}^{1}\right)$ denote the vector space of holomorphic differentials on $C$. Let $E$ be an elliptic curve. We denote the set of morphisms from $C$ to $E$ defined over $\mathbb{Q}$ by $\operatorname{Mor}_{\mathbb{Q}}(C, E)$ and give it the structure of an abelian group by defining the sum of two morphisms $\varphi_{1}$ and $\varphi_{2}$ to be the morphism which takes $x$ in $C$ to $\varphi_{1}(x)+\varphi_{2}(x)$ where + denotes addition in $E$.

Proposition 1. Consider the following four situations.

1. (quadratic twists) $E / \mathbb{Q}$ is an elliptic curve given by an equation $y^{2}=$ $a x^{3}+b x^{2}+c x+d$ and $D \in \mathbb{Z}[t]$ is a non-constant polynomial which is not $a$ perfect square in $\mathbb{C}[t] . C / \mathbb{Q}$ is a smooth, complete model of the curve defined by $s^{2}=D(t)$ and $E_{D} / \mathbb{Q}(t)$ is given by $D(t) y^{2}=a x^{3}+b x^{2}+c x+d$.

For each point $P=(x(t), y(t))$ in $E_{D}(\mathbb{Q}(t))$ we define an element $\varphi_{P}$ of $\operatorname{Mor}_{\mathbb{Q}}(C, E)$ by $\varphi_{P}(t, s)=(x(t), s y(t))$.

2. (cubic twists) $E / \mathbb{Q}$ is an elliptic curve given by an equation $y^{2}=x^{3}+k$ and $D \in \mathbb{Z}[t]$ is a non-constant polynomial which is not a perfect cube in $\mathbb{C}[t]$. $C / \mathbb{Q}$ is a smooth, complete model of the curve defined by $s^{3}=D(t)$ and $E_{D} / \mathbb{Q}(t)$ is given by $y^{2}=x^{3}+k(D(t))^{2}$.

For each point $P=(x(t), y(t))$ in $E_{D}(\mathbb{Q}(t))$ we define an element $\varphi_{P}$ of $\operatorname{Mor}_{\mathbb{Q}}(C, E)$ by $\varphi_{P}(t, s)=\left(x(t) s^{-2}, y(t) s^{-3}\right)$.

3. (quartic twists) $E / \mathbb{Q}$ is an elliptic curve given by an equation $y^{2}=x^{3}+a x$ and $D \in \mathbb{Z}[t]$ is a non-constant polynomial which is not a perfect square in $\mathbb{C}[t]$. $C / \mathbb{Q}$ is a smooth complete model of the curve defined by $s^{4}=D(t)$ and $E_{D} / \mathbb{Q}(t)$ is given by $y^{2}=x^{3}+a D(t) x$.

For each point $P=(x(t), y(t))$ in $E_{D}(\mathbb{Q}(t))$ we define an element $\varphi_{P}$ of $\operatorname{Mor}_{\mathbb{Q}}(C, E)$ by $\varphi_{P}(t, s)=\left(x(t) s^{-2}, y(t) s^{-3}\right)$.

4. (sextic twists) $E / \mathbb{Q}$ is an elliptic curve given by an equation $y^{2}=x^{3}+k$ and $D \in \mathbb{Z}[t]$ is a non-constant polynomial which is not a perfect square or a perfect cube in $\mathbb{C}[t] . C / \mathbb{Q}$ is a smooth, complete model of the curve defined by $s^{6}=D(t)$ and $E_{D} / \mathbb{Q}(t)$ is given by $y^{2}=x^{3}+k D(t)$.

For each point $P=(x(t), y(t))$ in $E_{D}(\mathbb{Q}(t))$ we define an element $\varphi_{P}$ of $\operatorname{Mor}_{\mathbb{Q}}(C, E)$ by $\varphi_{P}(t, s)=\left(x(t) s^{-2}, y(t) s^{-3}\right)$.

In each of the above four cases the map

$$
\lambda: E_{D}(\mathbb{Q}(t)) \rightarrow H^{0}\left(C, \Omega_{C / \mathbb{Q}}^{1}\right)
$$

given by

$$
\lambda(P)=\varphi_{P}^{*} \omega_{E},
$$

where $\varphi_{P}^{*} \omega_{E}$ denotes the pullback via $\varphi_{P}$ of the invariant differential $\omega_{E}$ on $E$, is a homomorphism with a finite kernel. 
Proof. We shall first prove that $\lambda$ is a homomorphism. Observe that $\lambda=\lambda_{2} \circ \lambda_{1}$ where $\lambda_{1}: E_{D}(\mathbb{Q}(t)) \rightarrow \operatorname{Mor}_{\mathbb{Q}}(C, E)$ by $\lambda_{1}(P)=\varphi_{P}$ and $\lambda_{2}: \operatorname{Mor}_{\mathbb{Q}}(C, E) \rightarrow$ $H^{0}\left(C, \Omega_{C / \mathbb{Q}}^{1}\right)$ by $\lambda_{2}(\varphi)=\varphi^{*} \omega_{E}$. In all four cases $\lambda_{1}$ is a homomorphism as may be verified by appealing to the addition law on $E$ and on $E_{D}$. Also $\lambda_{2}$ is a homomorphism as may be confirmed as in the proof of Theorem 5.2 of Chapter III of [42].

To see that $\lambda$ has a finite kernel notice that only constant morphisms yield a vanishing pullback of $\omega_{E}$. The result then follows in each case.

Observe that, by the proof of Proposition 1, polynomials $D$ for which the rank of $E_{D}(\mathbb{Q}(t))$ is large will have many independent morphisms from $C$ to $E$ of the appropriate form.

We shall use Proposition 1 to establish lower bounds for the rank of $E_{D}(\mathbb{Q}(t))$ for various curves $E_{D} / \mathbb{Q}(t)$ as above. To do so we shall start with a set of points in $E_{D}(\mathbb{Q}(t))$. Then it is a straightforward task to calculate the rank of the group generated by the image under $\lambda$ of these points in the vector space of holomorphic differentials on $C$. Since $\lambda$ is a homomorphism this rank is a lower bound for the rank of $E_{D}(\mathbb{Q}(t))$.

Let $D, n$ and $C$ be defined as at the start of this section. Let $\zeta_{n}$ be a primitive $n$th root of unity and define $\zeta$, an automorphism of $C$, by $\zeta(t, s)=$ $\left(t, \zeta_{n} s\right)$. Then $\zeta$ acts on the differentials of $C$ via the pullback $\zeta^{*} f(t, s) d t=$ $f\left(t, \zeta_{n} s\right) d t$ for any function $f$ on $C$. Hence one obtains a linear action on the space $H^{0}\left(C, \Omega_{C / \mathbb{Q}}^{1}\right)$ of holomorphic differentials on $C$. This action yields a decomposition

$$
H^{0}\left(C, \Omega_{C / \mathbb{Q}}^{1}\right)=\bigoplus_{i=0}^{n-1} H^{0}\left(C, \Omega_{C / \mathbb{Q}}^{1}\right)\left(\zeta_{n}^{i}\right),
$$

in which $H^{0}\left(C, \Omega_{C / \mathbb{Q}}^{1}\right)\left(\zeta_{n}^{i}\right)$ is the eigenspace on which $\zeta$ acts by multiplication by $\zeta_{n}^{i}$.

Corollary 1. Consider the four situations of Proposition 1. For each case we have

$$
\operatorname{rank} E_{D}(\mathbb{Q}(t)) \leq \operatorname{dim} H^{0}\left(C, \Omega_{C / \mathbb{Q}}^{1}\right)\left(\zeta_{n}\right)
$$

Proof. We check in each of the four cases that $\varphi_{P}^{*} \omega_{E}$ has the form $r(t) s d t$ where $r(t)$ is a rational function of $t$. Thus $\varphi_{P}^{*} \omega_{E}$ is in $H^{0}\left(C, \Omega_{C / \mathbb{Q}}^{1}\right)\left(\zeta_{n}\right)$ and our first assertion now follows from Proposition 1 since the kernel of $\lambda$ is finite.

We remark that the term on the right-hand side of the above inequality is equal to $\operatorname{Hom}_{\mathbb{Q}}(\operatorname{Jac}(C), E)$.

\section{POWER-FREE VALUES OF BINARY FORMS}

Let

$$
F(X, Y)=a_{r} X^{r}+a_{r-1} X^{r-1} Y+\cdots+a_{0} Y^{r}
$$


be a binary form with integer coefficients and positive degree $r$. Let $A, B, M$ and $k$ be integers with $M \geq 1$ and $k \geq 2$. We shall now define three counting functions associated with $k$-free values of $F$. We shall include the congruence condition $X \equiv A(\bmod M)$ and $Y \equiv B(\bmod M)$ in the definition of these functions since such a condition is required for applications involving the parity conjecture (see $\S 12$ ). It is also useful when one wishes to estimate the number of fields with discriminant in absolute value below a given bound and for which the ideal class group is of a certain type. Let $w$ be the largest positive integer such that $w^{k}$ divides $F(a, b)$ for all integers $a$ and $b$ with $a \equiv A(\bmod M)$ and $b \equiv B(\bmod M)$. For any real number $x$ let $N_{k}(x)$ and $P_{k}(x)$ denote the number of pairs of integers $(a, b)$ with $1 \leq a \leq x, 1 \leq b \leq x, a \equiv A(\bmod M)$ and $b \equiv B(\bmod M)$ for which $F(a, b)$ is $k$-free and for which $F(a, b) / w^{k}$ is $k$-free respectively. For any real number $x$ let $R_{k}(x)$ denote the number of $k$-free integers $t$ with $|t| \leq x$ for which there are integers $a$ and $b$ with $a \equiv A(\bmod M), b \equiv B(\bmod M)$ and $F(a, b)=t w^{k}$.

Let $f$ be a polynomial with integer coefficients which is irreducible over the rational numbers. Let $r$ denote the degree of $f$ and suppose that $r$ is at least 3. For any real number $x$ let $N(f, r-1, x)$ denote the number of positive integers $n$ not exceeding $x$ for which $f(n)$ is $(r-1)$-free. Hooley [19], [20] proved that

$$
N(f, r-1, x)=C_{7} x+O\left(x(\log x)^{(2 /(r+1))-1}\right),
$$

where $C_{7}$ is a non-negative number which depends on $f$ and $r$ and which is positive when $f$ has no fixed $(r-1)$ th power divisor larger than 1 . Let $F$ be a binary form as in (1). Suppose that $F$ has non-zero discriminant, $a_{r} a_{0} \neq 0$, and that all of the irreducible factors of $F$ over $\mathbb{Q}$ have degree at most 3 . Gouvêa and Mazur adapted the sieving argument of Hooley to prove that

$$
N_{2}(x)=C_{8} x^{2}+O\left(x^{2} /(\log x)^{\frac{1}{2}}\right),
$$

where $C_{8}$ is a non-negative number which depends on $A, B, M$ and $F$. To count the number of distinct quadratic twists of an elliptic curve given by $Y^{2}=$ $X^{3}+a X+b$ which their construction yields they need a corresponding result for $R_{2}(x)$. Suppose that $M$ is a positive integer, $a$ and $b$ are integers divisible by $M$ and that $4 a^{3}+27 b^{2} \neq 0$. Let $F(X, Y)=Y\left(X^{3}+a X Y^{2}+b Y^{3}\right)$ and let $A$ and $B$ be integers coprime with $M$. For any non-zero integer $h$ the number of pairs of integers $(s, t)$ with $F(s, t)=h$ is, since $Y$ is a factor of $F$, at most $3 \tau(h)$ where $\tau(h)$ denotes the number of positive integers which divide $h$. Using this fact and their result on $N_{2}(x)$ they deduce, in the special case above, that for each positive real number $\varepsilon$ there are positive numbers $C_{9}$ and $C_{10}$ which depend on $\varepsilon, a, b, A, B$ and $M$ such that if $x$ is a real number larger than $C_{9}$, then

$$
R_{2}(x)>C_{10} x^{\frac{1}{2}-\varepsilon}
$$

In a recent article Greaves [16] improved on the result (2) of Gouvêa and Mazur. Let $F, A, B, M$ and $k$ be as in the introduction to this section. Define $w_{0}$ to be the largest positive integer such that $w_{0}^{k}$ divides $F(a, b)$ for all integers $a$ and $b$. Let $m$ denote the degree of the largest irreducible factor 
of $F$ over $\mathbb{Q}$. Greaves assumes that $F$ has non-zero discriminant, $a_{r} a_{0} \neq 0$ and $w_{0}=1$. He then proves that there is a non-negative number $C_{11}$, which depends on $M, A, B$ and $F$, such that if $] m \leq 6$, then

$$
N_{2}(x)=C_{11} x^{2}+O\left(x^{2} /(\log x)^{\frac{1}{3}}\right)
$$

and there is a non-negative number $C_{12}$, which depends on $M, A, B, F$ and $k$, such that if $m \leq 2 k+1$, then

$$
N_{k}(x)=C_{12} x^{2}+O\left(x^{2} / \log x\right)
$$

here the $O$-constants depend only on $F$. Further he proves that if $M=1$, then $C_{11}$ and $C_{12}$ are positive.

Our aim is to prove corresponding results for $R_{k}(x)$. As a first step we shall modify the arguments of Greaves so that they apply to the functions $P_{k}(x)$. Again let $F, A, B, M$ and $k$ be as in the introduction to this section. For each prime $p$ let $\operatorname{ord}_{p} t$ denote the $p$-adic order of $t$. Put $m=\operatorname{ord}_{p} M, v=\operatorname{ord}_{p} w^{k}$ and $l=k+m+v$. Let $S$ be the set of those primes which divide $M, w$, or the discriminant of $F$. Let $s(p)$ denote the number of pairs of integers $(a, b)$ with $1 \leq a \leq p^{l}, 1 \leq b \leq p^{l}, a \equiv A\left(\bmod p^{m}\right), b \equiv B\left(\bmod p^{m}\right)$ and for which $F(a, b) \not 0\left(\bmod p^{k+v}\right)$. Put

$$
C_{13}=\prod_{p} \frac{s(p)}{p^{2 l}}
$$

(The authors would like to thank Professor M. Filaseta for his comments concerning the definition of $C_{13}$.) The number of pairs $(a, b)$ with $1 \leq a \leq p^{k}$ and $1 \leq b \leq p^{k}$ for which $p$ divides both $a$ and $b$ is $p^{2 k-2}$. Further the number of pairs $(a, b)$ with $1 \leq a \leq p^{k}, 1 \leq b \leq p^{k}$, for which $p$ does not divide both $a$ and $b$ and for which $F(a, b) \equiv 0\left(\bmod p^{k}\right)$, is at most $r p^{k}$ provided that $p$ does not divide the discriminant of $F$ (see, for example, Theorem 2 of [43]). Thus

$$
s(p) \geq p^{2 k}-p^{2 k-2}-r p^{k}
$$

for primes $p$ which are not in $S$. It follows from the definition of $w$ that $s(p) \geq 1$ for all primes $p$. Therefore the product defining $C_{13}$ converges to a positive real number.

Lemma 2. Let $A, B, M, F, k$ and $w$ be defined as in the introduction to this section. Suppose that $F$ has non-zero discriminant, $a_{r} a_{0} \neq 0$ and that $m$ denotes the degree of the largest irreducible factor of $F$ over $\mathbb{Q}$. If $m \leq 6$, then

$$
P_{2}(x)=C_{13} x^{2}+O\left(x^{2} /(\log x)^{\frac{1}{3}}\right)
$$

while if $m \leq 2 k+1$, then

$$
P_{k}(x)=C_{13} x^{2}+O\left(x^{2} / \log x\right),
$$

where the $O$-constants depend only on $F, M$ and $k$.

Proof. Put $\lambda=(\log x) / 2 k$ and assume that $x$ is sufficiently large that

$$
\prod_{p \leq \lambda} p^{l} \leq x^{\frac{2}{3}},
$$


that all primes $p$ in $S$ are less than $\lambda$ and that all primes which divide $a_{r} a_{0}$ are also less than $\lambda$.

We suppose that $F(X, Y)=\prod_{i=1}^{t} F_{i}(X, Y)$ where the forms $F_{i}$ have integer coefficients and are irreducible in $\mathbb{Q}[X, Y]$. Let $E_{0}(x)$ denote the number of pairs of integers $(a, b)$ with $1 \leq a \leq x$ and $1 \leq b \leq x$ for which $p$ divides both $a$ and $b$ for some prime $p$ with $p>\lambda$. For $i=1, \ldots, t$ let $E_{i}(x)$ denote the number of pairs $(a, b)$ with $1 \leq a \leq x$ and $1 \leq b \leq x$ for which there is a prime $p$ larger than $\lambda$ which does not divide both $a$ and $b$ and for which $p^{k}$ divides $F_{i}(a, b)$. Put

$$
V=\prod_{p \leq \lambda} p^{l}
$$

and for integers $i$ and $j$ denote the set of pairs of integers $(a, b)$ with $i V<$ $a \leq(i+1) V$ and $j V<b \leq(j+1) V$ by $W_{i, j}$. Notice that the set of pairs $(a, b)$ with $1 \leq a \leq x$ and $1 \leq b \leq x$ contains the union of the sets $W_{i, j}$ with $0 \leq i, j \leq\left[\frac{x}{V}\right]-1$ and is contained in the union of these sets with $0 \leq i, j \leq\left[\frac{x}{V}\right]$. Thus, by the Chinese Remainder Theorem, and (5),

$$
P_{k}(x)=\left(\prod_{p \leq \lambda} \frac{s(p)}{p^{2 l}}\right) x^{2}+O\left(x^{\frac{5}{3}}\right)+O\left(\sum_{i=0}^{t} E_{i}(x)\right)
$$

and so, by (4),

$$
P_{k}(x)=C_{13} x^{2}+O\left(x^{2} / \log x\right)+O\left(\sum_{i=0}^{t} E_{i}(x)\right)
$$

here the $O$-constants depend on $F, M$ and $k$.

It only remains to estimate the error terms $E_{i}(x)$ for $i=0, \ldots, t$. This is done in [16] by Greaves with $\lambda=\frac{1}{3} \log x$. Moreover Greaves' argument works equally well with $\lambda=(\log x) / 2 k$; only a minor modification is required in the proof of Lemma 2 of [16] where the terms $E_{i}^{(1)}(x)$ are estimated. The result now follows.

Let $F$ be a binary form, as in (1), with integer coefficients, non-vanishing discriminant, degree $r$ at least 3 and $a_{r} a_{0} \neq 0$. For any integer $x$ let $R(x)$ denote the number of integers $t$ with $|t| \leq x$ for which there exist integers $a$ and $b$ with $F(a, b)=t$. In 1938 Erdös and Mahler [11] proved that there is a positive number $C_{14}$, which depends on $F$, such that for $x$ sufficiently large

$$
R(x)>C_{14} x^{\frac{2}{r}}
$$

Mahler [27] has shown that this estimate is best possible apart from the number $C_{14}$. We shall modify the argument of Erdös and Mahler in order to estimate the number of distinct $k$-free integers $t$ of absolute value at most $x$ for which there are integers $a$ and $b$ with $F(a, b)=t$.

Theorem 1. Let $A, B, M$ and $k$ be integers with $M \geq 1$ and $k \geq 2$. Let $F$, as in (1), be a binary form with integer coefficients, non-zero discriminant and degree $r$ with $r \geq 3$. Let $m$ be the largest degree of an irreducible factor of $F$ 
over $\mathbb{Q}$ and suppose that $m \leq 2 k+1$ or that $k=2$ and $m=6$. There are positive numbers $C_{15}$ and $C_{16}$ which depend on $M, k$ and $F$, such that if $x$ is a real number larger than $C_{15}$, then

$$
R_{k}(x)>C_{16} x^{\frac{2}{f}}
$$

Erdös and Mahler appealed to a modification of Mahler's [26] $p$-adic version of the Thue-Siegel theorem to establish their result. We have found it convenient to appeal to a recent result of Stewart [43] on the number of solutions of the Thue equation for the proof of Theorem 1, although we could also get by with Mahler's result or Lemma 8 of [11]. In fact the full power of these results is not needed since in the proof of Theorem 1 we only need an estimate for relatively small solutions of the Thue equations involved and an argument based on a gap principle suffices for this purpose. Thus the Thue-Siegel principle (see [43]) is not required.

\section{Proof of Theorem 1}

We may assume that $k \leq r$ since $m$ is at most $r$ and since if an integer is $k$-free it is also $(k+1)$-free. We may also assume, without loss of generality, that $a_{r} a_{0} \neq 0$. To see this note that if $a_{r} a_{0}=0$, then there is a matrix

$$
\mathscr{M}=\left(\begin{array}{ll}
m_{1} & m_{2} \\
m_{3} & m_{4}
\end{array}\right)
$$

in $S L(2, \mathbb{Z})$ for which $F\left(m_{1} X+m_{2} Y, m_{3} X+m_{4} Y\right)=a_{r}^{\prime} X^{r}+a_{r-1}^{\prime} X^{r-1} Y+$ $\cdots+a_{0}^{\prime} Y^{r}$ with $a_{r}^{\prime} a_{0}^{\prime} \neq 0$. Denote $F\left(m_{1} X+m_{2} Y, m_{3} X+m_{4} Y\right)$ by $F_{\mathscr{M}}(X, Y)$. Since $\mathscr{M}$ is in $S L(2, \mathbb{Z})$ the discriminant of $F_{\mathscr{M}}$ is the same as the discriminant of $F$. Further let $A^{\prime}=m_{1} A+m_{2} B$ and $B^{\prime}=m_{3} A+m_{4} B$ and observe that the set of integers of the form $F_{M}(a, b)$ with $a \equiv A^{\prime}(\bmod M)$ and $b \equiv B^{\prime}(\bmod M)$ is the same as the set of integers of the form $F(a, b)$ with $a \equiv A(\bmod M)$ and $b \equiv B(\bmod M)$. Accordingly, we may replace $F$ by $F_{\mathscr{M}}$ if necessary.

For any prime $p$ and any rational number $a$ let $|a|_{p}$ denote the $p$-adic value of $a$, normalized so that $|p|_{p}=p^{-1}$. Let $u$ be a positive real number. For any real number $\theta$ with $0<\theta \leq 1$ and for any non-zero integer $h$ we define $s(h)$ by

$$
s(h)=\prod_{\substack{p \leq u^{\theta} \\|h|_{p}^{-1} \leq u^{\theta} \\ p \nmid w D}}|h|_{p}^{-1},
$$

where $D$ denotes the discriminant of $F$. Let $U$ denote the set of pairs of integers $(a, b)$ with $1 \leq a \leq u, 1 \leq b \leq u$ and $F(a, b) \neq 0$ and such that the only primes which divide the greatest common divisor of $a$ and $b$ also divide $w D$. Next we define $S(\theta, u)$ by

$$
S(\theta, u)=\prod_{(a, b) \in U} s(F(a, b))
$$


We shall now estimate $S(\theta, u)$ by an argument similar to that of Lemma 1 of [11]. Note, however, that the proof of Lemma 1 of [11] is incorrect as it stands since the authors do not give a valid estimate for $\operatorname{ord}_{p} G$. They overlook the contribution from pairs $(x, y)$ for which $p$ divides both $x$ and $y$. This can be easily fixed by requiring that the pairs $(x, y)$ in the product defining $G$ are pairs of coprime integers. The balance of their argument goes through unchanged.

Let $p$ be a prime which does not divide $w D$ and let $z$ be a positive integer for which $p^{z} \leq u^{\theta}$. Note that the content of $F$ divides $D$. For each integer $b$ which is coprime with $p$ there are at most $r$ integers $a$ modulo $p^{z}$ for which

$$
F(a, b) \equiv 0 \quad\left(\bmod p^{z}\right) .
$$

Thus there are at most $r u\left(\left[\frac{u}{p^{2}}\right]+1\right)$ solutions of $(6)$ from $U$ for which $p$ does not divide $b$. The same estimate applies for the number of solutions of (6) from $U$ for which $p$ does not divide $a$. Thus

$$
\operatorname{ord}_{p} S(\theta, u) \leq \sum_{z=1}^{\left[\frac{\theta \log u}{\log p}\right]} \frac{4 r u^{2}}{p^{z}} \leq \sum_{z=1}^{\infty} \frac{4 r u^{2}}{p^{z}}=\frac{4 r u^{2}}{p-1}
$$

Therefore

$$
S(\theta, u) \leq \exp \left(4 r u^{2} \sum_{\substack{p \leq u^{\theta} \\ p \nmid w D}} \frac{\log p}{p-1}\right) .
$$

Hence, by Theorem 425 of [17], there is a positive number $C_{17}$, which depends on $\theta$, such that if $u$ exceeds $C_{17}$, then

$$
S(\theta, u) \leq u^{5 \theta r u^{2}} .
$$

Consequently, if $u$ exceeds $C_{17}$, the number of pairs $(a, b)$ in $U$ for which $s(F(a, b)) \geq u^{\frac{1}{8}}$ is at most

$$
40 \theta r u^{2} \text {. }
$$

Next observe, as in Lemma 2 of [11], that if $h$ and $b$ are integers with $|h| \leq u^{\frac{1}{2}}$ and $1 \leq b \leq u$, then there are at most $r$ integers $a$ with $F(a, b)=h$. Accordingly, the number of pairs of integers $(a, b)$ with $1 \leq a \leq u, 1 \leq b \leq u$ and $|F(a, b)| \leq w^{r} u^{\frac{1}{2}}$ is at most

$$
3 r w^{r} u^{\frac{3}{2}}
$$

We now fix $\theta$ by putting

$$
\theta=C_{13} / 120 r
$$

recall (3). Let $T$ be the set of pairs of integers $(a, b)$ with $1 \leq a \leq u$, $1 \leq b \leq u, a \equiv A(\bmod M), b \equiv B(\bmod M)$ and for which $F(a, b) / w^{k}$ is $k$-free, $|F(a, b)| \geq w^{r} u^{\frac{1}{2}}$ and $s(F(a, b))<u^{\frac{1}{8}}$. Note that if $F(a, b) / w^{k}$ is $k$-free, then, since $k \leq r$, the greatest common divisor of $a$ and $b$ divides $w$. 
Thus $T$ is contained in $U$. For any set $X$ we denote the cardinality of $X$ by $|X|$. By Lemma 2, (7), (8) and (9) there are positive numbers $C_{18}$ and $C_{19}$, which depend on $F, M$ and $k$, such that if $u$ exceeds $C_{18}$, then

$$
|T|>\frac{1}{2} C_{13} u^{2}
$$

As we remarked above, if $(a, b)$ is in $T$, then the greatest common divisor of $a$ and $b$ divides $w$. Let $d$ be that divisor of $w$ which occurs most often as the greatest common divisor of a pair $(a, b)$ from $T$. Let $T_{0}$ be the set of pairs $(a, b)$ from $T$ whose greatest common divisor is $d$ and let $T_{1}$ be the set of pairs $\left(\frac{a}{d}, \frac{b}{d}\right)$ with $(a, b)$ in $T_{0}$. It follows from (10) that

$$
\left|T_{1}\right|>C_{13} u^{2} /(2 \tau(w)) \text {, }
$$

provided that $u$ exceeds $C_{18}$. Observe that if $\left(a^{\prime}, b^{\prime}\right)$ is in $T_{1}$, then $a^{\prime}$ and $b^{\prime}$ are coprime, $1 \leq a^{\prime} \leq u, 1 \leq b^{\prime} \leq u,\left|F\left(a^{\prime}, b^{\prime}\right)\right| \geq u^{\frac{1}{2}}$ and $s\left(F\left(a^{\prime}, b^{\prime}\right)\right)<u^{\frac{1}{8}}$.

For any non-zero integer $h$ let $\omega(h)$ denote the number of distinct prime factors of $h$. Let $h$ be an integer for which there is a pair $\left(a^{\prime}, b^{\prime}\right)$ in $T_{1}$ with

$$
F\left(a^{\prime}, b^{\prime}\right)=h \text {. }
$$

Put $h=s\left(F\left(a^{\prime}, b^{\prime}\right)\right) \cdot g$ and note that $|g| \geq|h|^{\frac{3}{4}}$. If $u$ is larger than $|D|^{24}$, then $h$ is larger than $|D|^{12}$ and so, on taking $\varepsilon=1 / 12$ in Corollary 1 of [43], we deduce that the number of pairs $\left(a^{\prime}, b^{\prime}\right)$ from $T_{1}$ for which (12) holds is at most

$$
5600 r^{1+\omega(g)} \text {. }
$$

Let $H$ denote the maximum of $\left\{\left|a_{0}\right|, \ldots,\left|a_{r}\right|\right\}$ and observe that if $\left(a^{\prime}, b^{\prime}\right)$ is in $T_{1}$, then

$$
\left|F\left(a^{\prime}, b^{\prime}\right)\right| \leq r H u^{r}
$$

The prime divisors $p$ of $g$ either divide $w D$ or satisfy $\left|F\left(a^{\prime}, b^{\prime}\right)\right|_{p}^{-1} \geq u^{\theta}$. Thus, provided that $u^{\theta} \geq r H$,

$$
\omega(g) \leq \omega(w D)+(r+1) / \theta .
$$

Therefore, by (9), (11), (13) and (15) there are positive numbers $C_{20}$ and $C_{21}$, which depend on $F, M$ and $k$, such that if $u$ exceeds $C_{20}$, then the number of distinct integers $h$ of the form $F\left(a^{\prime}, b^{\prime}\right)$ with $\left(a^{\prime}, b^{\prime}\right)$ in $T_{1}$ is at least $C_{21} u^{2}$ and so the number of distinct integers $F(a, b)$ with $(a, b)$ in $T$ is also at least $C_{21} u^{2}$.

Let $x$ be a real number with $x>r H C_{20}^{r}$ and put $u=(x / r H)^{\frac{1}{r}}$; note that $u$ exceeds $C_{20}$. We now define $T$ as above and observe that if $(a, b)$ is in $T$, then $|F(a, b)| \leq x$. Thus

$$
R_{k}(x) \geq|T| \geq C_{21}(x / r H)^{\frac{2}{r}},
$$

and this completes the proof of Theorem 1 . 


\section{VALUES OF BINARY FORMS MODULO $k$ TH POWERS}

Let $A, B, M$ and $k$ be integers with $M \geq 1$ and $k \geq 2$. Let $F$ be a binary form as in (1). For any real number $x$ let $S_{k}(x)$ denote the number of $k$-free integers $t$ with $|t| \leq x$ for which there exist integers $a, b$ and $z$ with $a \equiv A(\bmod M), b \equiv B(\bmod M), z$ non-zero and $F(a, b)=t z^{k}$. It is the function $S_{k}(x)$ which we use to count the number of different twists of elliptic curves produced by our constructions. Plainly we have

$$
S_{k}(x) \geq R_{k}(x)
$$

and so the estimate for $R_{k}(x)$ given by Theorem 1 furnishes a lower bound for $S_{k}(x)$ provided that the hypotheses of Theorem 1 apply. Our next result gives a weaker lower bound for $S_{k}(x)$ which, however, is more widely applicable.

Our first version of this result gave a lower bound for $S_{k}(x)$ of $C_{23} x^{1 / r} / \log x$. Professor A. Granville pointed out to us how to improve this to $C_{23} x^{2 / r} /(\log x)^{2}$. We are very grateful to him for allowing us to incorporate his ideas into our proof.

Theorem 2. Let $A, B, M$ and $k$ be integers with $M \geq 1$ and $k \geq 2$. Let $F$ be a binary form with integer coefficients and degree $r$ which is not a constant multiple of a power of a linear form and which is not divisible over $\mathbb{Q}$ by the kth power of a non-constant binary form. There are positive numbers $C_{22}$ and $C_{23}$, which depend on $F$ and $M$, such that if $x$ is a real number.larger than $C_{22}$, then

$$
S_{k}(x)>C_{23} x^{\frac{2}{r}} /(\log x)^{2}
$$

Proof. $C_{24}, C_{25}, \ldots$ will denote positive numbers which are effectively computable in terms of $F$ and $M$. We may write $F(X, Y)=F_{1}(X, Y) \cdots F_{l}(X, Y)$ where $F_{1}, \ldots, F_{l}$ are binary forms with integer coefficients, $F_{i+1}$ divides $F_{i}$ for $i=1, \ldots, l-1$ and $F_{i}$ is either linear or has a non-zero discriminant for $i=1, \ldots, l$. Since $F$ is not divisible by the $k$ th power of a non-constant binary form, $l$ is at most $k-1$. Next we write $F_{1}(X, Y)=$ $G_{1}(X, Y) \cdots G_{m}(X, Y)$ where $G_{1}, \ldots, G_{m}$ are non-constant forms with integer coefficients which are irreducible in $\mathbb{Q}[X, Y]$.

We first deal with the case that one of $G_{1}, \ldots, G_{m}$ is non-linear. We may assume without loss of generality that $G_{1}$ is non-linear. Put $G(X)=G_{1}(X, 1)$ for brevity.

Let $u$ be a positive real number for which $u / 2$ exceeds the maximum of $M$, the content of $F$ and the discriminant of $F_{1}$. Let $W$ be the set of primes $p$ with $u / 2<p<u$ for which the congruence $G(X) \equiv 0(\bmod p)$ has a solution. By the Chebotarev density theorem [24] there are positive numbers $C_{24}$ and $C_{25}$ such that if $u$ exceeds $C_{24}$, then

$$
|W|>C_{25} u / \log u \text {. }
$$

Let $p$ be a prime in $W$ and let $v$ be an integer with $0 \leq v<p$ for which $G(v) \equiv 0(\bmod p)$, hence for which $F_{1}(v, 1) \equiv 0(\bmod p)$. Since $p$ is larger than $u / 2, p$ does not divide the content or the discriminant of $F_{1}$. Thus, by Hensel's lemma, there is a unique integer $v_{1}$ with $0 \leq v_{1}<p$ such that 
if $x \equiv v(\bmod p)$ and $F_{1}(x, 1) \equiv 0\left(\bmod p^{2}\right)$, then $x \equiv v+v_{1} p\left(\bmod p^{2}\right)$. We have $F_{1}(r, s) \equiv 0(\bmod p)$ whenever $(r, s)$ is a pair of integers with $r \equiv v s(\bmod p)$. The set of such pairs $(r, s)$ forms a lattice $\Lambda_{p}$ of determinant $p$. Further the set of pairs $(r, s)$ in $\Lambda_{p}$ for which $F_{1}(r, s) \equiv 0\left(\bmod p^{2}\right)$ forms a sublattice of determinant $p^{2}$.

Let $\left(r_{0}, s_{0}\right)$ be an element of $\Lambda_{p}$ different from $(0,0)$ for which $\max \left(\left|r_{0}\right|,\left|s_{0}\right|\right)$ is minimal and put $m_{p}=\max \left(\left|r_{0}\right|,\left|s_{0}\right|\right)$. Let $d$ denote the degree of $G_{1}$. There exists a positive number $C_{26}$ such that whenever $(r, s)$ is in $\Lambda_{p}$ with $(r, s) \neq(0,0)$, then

$$
0<\left|G_{1}(r, s)\right|<C_{26}(\max (|r|,|s|))^{d} ;
$$

note that $G_{1}(r, s)$ is non-zero since $G_{1}$ is irreducible and of degree at least two. Thus if $u$ exceeds $C_{27}$ and the maximum of $|r|$ and $|s|$ is at most $u^{\frac{1}{2}}$, then at most $d$ primes from $W$ divide $G_{1}(r, s)$. If $u$ exceeds $C_{28}$ the number of pairs of integers $(r, s)$ with

$$
\max (|r|,|s|)<\left(C_{25} u /(10 d \log u)\right)^{\frac{1}{2}}
$$

is at most $C_{25} u /(2 d \log u)$. Accordingly, by inequality (16), there are at most $C_{25} u /(2 \log u)$ primes $p$ in $W$ for which $m_{p}$ is less than $\left(C_{25} u /(10 d \log u)\right)^{\frac{1}{2}}$. Let $W_{1}$ be the set of primes $p$ in $W$ for which

$$
m_{p} \geq\left(C_{25} u /(10 d \log u)\right)^{\frac{1}{2}},
$$

and note that

$$
\left|W_{1}\right| \geq C_{25} u /(2 \log u) .
$$

Let $p$ be a prime in $W_{1}$ and let $\left(r_{0}, s_{0}\right)$ and $\left(r_{1}, s_{1}\right)$ be a basis for $\Lambda_{p}$ with $\max \left(\left|r_{1}\right|,\left|s_{1}\right|\right)$ minimal. It follows from (17), as in Lemma 1 of [16] (see in particular (2.8) of [16]), that

$$
\max \left(\left|r_{0}\right|,\left|s_{0}\right|,\left|r_{1}\right|,\left|s_{1}\right|\right) \leq C_{29}(u \log u)^{\frac{1}{2}} .
$$

Since $M$ and $p$ are coprime and the determinant $\left|r_{0} s_{1}-r_{1} s_{0}\right|$ of $\Lambda_{p}$ is $p$, there exist integers $j$ and $k$ with $0 \leq j<M$ and $0 \leq k<M$ for which $j r_{0}+k r_{1} \equiv A(\bmod M)$ and $j s_{0}+k s_{1} \equiv B(\bmod M)$. Put $\left(r_{2}, s_{2}\right)=j\left(r_{0}, s_{0}\right)+$ $k\left(r_{1}, s_{1}\right),\left(r_{3}, s_{3}\right)=\left(r_{2}, s_{2}\right)+M\left(r_{0}, s_{0}\right)$ and $\left(r_{4}, s_{4}\right)=\left(r_{2}, s_{2}\right)+M\left(r_{1}, s_{1}\right)$. Note that $r_{i} \equiv A(\bmod M), s_{i} \equiv B(\bmod M)$ and $F_{1}\left(r_{i}, s_{i}\right) \equiv 0(\bmod p)$ for $i=2,3$ and 4 . Further, we can find two linearly independent vectors in $\left\{\left(r_{2}, s_{2}\right),\left(r_{3}, s_{3}\right),\left(r_{4}, s_{4}\right)\right\}$ and these two vectors generate a sublattice of $\Lambda_{p}$ of determinant at most $C_{30} u \log u$ by virtue of (19). Since the set of pairs $(r, s)$ in $\Lambda_{p}$ for which $F_{1}(r, s) \equiv 0\left(\bmod p^{2}\right)$ forms a sublattice of $\Lambda_{p}$ of determinant $p^{2}$ and $p^{2}$ exceeds $u^{2} / 4$ we see that $\operatorname{ord}_{p} F_{1}\left(r_{i}, s_{i}\right)=1$ when $i$ is 2,3 or 4 provided that $u$ exceeds $C_{31}$. Thus for each prime $p$ in $W_{1}$ there exists a pair of integers $(a, b)$ with

$$
a \equiv A \quad(\bmod M), \quad b \equiv B \quad(\bmod M), \quad \operatorname{ord}_{p} F_{1}(a, b)=1
$$


and

$$
\max (|a|,|b|) \leq C_{32}(u \log u)^{\frac{1}{2}} .
$$

Assume that $F$ is as in (1) and put $H=\max \left\{\left|a_{r}\right|, \ldots,\left|a_{0}\right|\right\}$. Then put $u=(x / r H)^{\frac{2}{r}} /\left(C_{32}^{2} \log x\right)$ so that when $x$ exceeds $C_{33}$ and (21) holds, then $|F(a, b)| \leq x$. Observe that, by (18),

$$
\left|W_{1}\right|>C_{34} x^{\frac{2}{r}} /(\log x)^{2}
$$

To each prime $p$ in $W_{1}$ there exists a pair of integers $(a, b)$ satisfying (20) and with $|F(a, b)| \leq x$. Put $F(a, b)=t_{p} z^{k}$ where $t_{p}$ and $z$ are integers with $t_{p} k$-free and $z$ positive. Next put $T=\left\{t_{p} \mid p \in W_{1}\right\}$. Since $l$ is less than $k$, we have

$$
1 \leq \operatorname{ord}_{p} F(a, b)<k,
$$

and therefore $p$ divides $t_{p}$ for each prime $p$ in $W_{1}$. Further for each integer $t$ in $T$ we have $|t| \leq x$. Furthermore, for each integer $t$ in $T$ there are at most $r$ primes $p$ from $W_{1}$ with $t_{p}=t$ provided that $u$ exceeds $C_{35}$, since each prime $p$ in $W_{1}$ exceeds $u / 2$. Thus

$$
S_{k}(x) \geq|T| \geq\left|W_{1}\right| / r,
$$

and the result follows from (22).

We are left with the case where $G_{1}, \ldots, G_{m}$ are linear forms. We first treat the case when $m$ is at least three. As before let $w$ be the largest integer such that $w^{2}$ divides $F_{1}(a, b)$ for all integers $a$ and $b$ with $a \equiv A(\bmod M)$ and $b \equiv B(\bmod M)$. Let $u$ be a positive real number and define $T$ to be the set of integers $F_{1}(a, b)$ with $1 \leq a \leq u, 1 \leq b \leq u, a \equiv A(\bmod M), b \equiv$ $B(\bmod M)$ and $F_{1}(a, b) / w^{2}$ square-free. It is established in the penultimate paragraph of the proof of Theorem 1 that there exists a positive number $C_{36}$ such that if $u$ exceeds $C_{36}$, then $|T|$ is at least $C_{37} u^{2}$. If $F_{1}(a, b)$ is in $T$ and $p$ is a prime which divides $F_{1}(a, b)$ but does not divide $w$, then

$$
1 \leq \operatorname{ord}_{p} F(a, b)<k \text {. }
$$

Put $u=(x / r H)^{\frac{1}{r}}$ and note that $|F(a, b)|$ is at most $x$ when the maximum of $|a|$ and $|b|$ is at most $u$. Therefore

$$
S_{k}(x) \geq|T| / 2^{\omega(w)} \geq C_{38} x^{\frac{2}{r}}
$$

when $x$ is at least $C_{39}$.

Finally we must deal with the possibility that $F_{1}$ is the product of two linear forms. We may suppose that $F_{1}(X, Y)=(c X+d Y)(e X+f Y)$ with $c, d, e$, and $f$ integers. Since $c X+d Y$ and $e X+f Y$ are non-proportional, we may assume that $c$ is non-zero. Put $a=A+k M$ and $b=B+l c M$ so that

$$
F_{1}(a, b)=(c A+d B+(k+d l) c M)(e A+f B+(e k+c f l) M) .
$$

Let $S_{1}$ denote the greatest common divisor of $c A+d B$ and $c M$. Then by the prime number theorem for arithmetic progressions with error term, if $u$ 
is a real number larger than $C_{40}$, then the number of primes $p$ of the form $\left((c A+d B) / S_{1}+t\left(c M / S_{1}\right)\right)$ with $t$ an integer and $u / 2<p<u$ is at least $C_{41} u / \log u$. For each integer $t$ as above we put $k=t-d l$ so that we have $(e A+f B+(e k+c f l) M)=(e A+f B+e t M+(c f-d e) l M)$. Let $S_{2}$ be the greatest common divisor of $e A+f B+e t M$ and $(c f-d e) M$. Then, again by the prime number theorem for arithmetic progressions with error term, if $u$ is a real number larger than $C_{42}$, then the number of primes $p$ of the form

$$
\left((e A+f B+e t M) / S_{2}+\left((c f-d e) M / S_{2}\right) l\right)
$$

with $l$ an integer and $u<p<2 u$ is at least $C_{43} u / \log u$. Therefore when $u$ exceeds $C_{44}$, there exist at least $C_{45}(u / \log u)^{2}$ pairs of primes $(p, q)$ with $u / 2<p<u$ and $u<q<2 u$ for which there exist integers $a(=a(p, q))$ and $b(=b(p, q))$ with $a \equiv A(\bmod M), b \equiv B(\bmod M), 0<|c a+d b| \leq|c| M u$, $0<|e a+f b| \leq 2|c f-d e| M u$ and for which $p$ divides $c a+d b$ and $q$ divides $e a+f b$. For each pair of primes $(p, q)$ as above we put $t_{(p, q)}=$ $F(a(p, q), b(p, q))$. We now take

$$
u=x^{\frac{1}{r}} /(\max (|c| M, 2|c f-d e| M)) \text {. }
$$

Plainly $\left|t_{(p, q)}\right|$ is at most $x$ for all pairs $(p, q)$ and provided that $x$ exceeds $C_{45}$ each integer $t_{(p, q)}$ contributes 1 to $S_{k}(x)$. Our result now follows.

\section{QUADRATIC TWISTS}

In the next four sections we shall establish unconditional estimates for the number of twists of large rank of various elliptic curves. For many of our calculations in these sections we have employed the symbolic computation package MAPLE.

Our first result gives an unconditional analogue of the result of Gouvêa and Mazur [15] but with an exponent of $\frac{1}{7}$ in place of $\frac{1}{2}$. The proof depends on a construction used by Mestre [30] to prove that there are infinitely many elliptic curves over $\mathbb{Q}$ with given modular invariant and rank at least 2 . Our contribution will be to make this result quantitative.

Theorem 3. Let $a$ and $b$ be rational numbers and assume $a b\left(4 a^{3}+27 b^{2}\right) \neq 0$. There exist positive numbers $C_{46}$ and $C_{47}$, which depend on $a$ and $b$, such that if $T$ is a real number larger than $C_{46}$, then the number of square-free integers $d$ with $|d| \leq T$ for which the curve given by

$$
d y^{2}=x^{3}+a x+b
$$

has rank at least 2 is at least $C_{47} T^{\frac{1}{7}} /(\log T)^{2}$.

Proof. Following Mestre we put

$$
f(t)=t^{3}+a t+b, \quad g(t)=\left(-\frac{b}{a}\right)\left(\left(t^{4}+t^{2}+1\right) /\left(t^{4}+t^{2}\right)\right)
$$

and

$$
D(t)=a^{4}\left(t^{2}+1\right)^{4} t^{6} f(g(t))
$$


Let $P_{1}=\left(g(t),\left(a^{2}\left(t^{2}+1\right)^{2} t^{3}\right)^{-1}\right)$ and $P_{2}=\left(t^{2} g(t),\left(a\left(t^{2}+1\right)\right)^{-2}\right)$ and note that $P_{1}$ and $P_{2}$ are points on $E_{D}$ where $E_{D}$ is given by the equation $D(t) y^{2}=$ $x^{3}+a x+b$. Let $E$ be the curve given by $y^{2}=x^{3}+a x+b$. The invariant differential $\omega_{E}$ on $E$ is given by $\omega_{E}=\frac{d x}{2 y}$. In the notation of Proposition $1, \varphi_{P_{1}}^{*} \omega_{E}=a b\left(2 t^{2}+1\right) \frac{d t}{s}$ and $\varphi_{P_{2}}^{*} \omega_{E}=-a b\left(t^{5}+2 t^{3}\right) \frac{d t}{s}$ and so the rank of $E_{D}(\mathbb{Q}(t))$ is at least 2. The degree of $D$ is 14 and $D$ is divisible by $t^{2}+1$ in $\mathbb{Q}[t]$. Provided that $a b \neq 0,\left(t^{2}+1\right)^{2}$ does not divide $D$ in $\mathbb{Q}[t]$. Put $F(X, Y)=Y^{14} D(X / Y)$. Let $F_{1}$ and $F_{2}$ be binary forms satisfying $F=F_{1} F_{2}^{2}$ with $F_{2}$ in $\mathbb{Z}[X, Y]$ and of maximal degree. Then $X^{2}+Y^{2}$ divides $F_{1}$ and we may apply Lemma 1 and Theorem 2 to give our result.

Our next two theorems treat quadratic twists of special families of elliptic curves; for these families we are able to establish larger exponents than $\frac{1}{7}$.

Theorem 4. Let $a, b$ and $c$ be rational numbers and put $A=-\left(a^{2}+a c+c^{2}\right)$. Assume that $a$ and $c$ are not both zero and that $4 A^{3}+27 b^{2} \neq 0$. There exist positive numbers $C_{48}$ and $C_{49}$, which depend on $a, b$ and $c$, such that if $T$ is a real number larger than $C_{48}$, then the number of square-free integers $d$ with $|d| \leq T$ for which the curve given by

$$
d y^{2}=x^{3}+A x+b
$$

has rank at least 2 is at least $C_{49} T^{\frac{1}{4}}$.

Proof. Motivated by the construction of Schoof, $\S 2$ of [37] (see also [10], [29]), we put

$$
r_{1}(t)=-c t^{2}+2 a t+a+c, \quad r_{2}(t)=(a+c) t^{2}+2 c t-a
$$

and

$$
D(t)=\left(r_{1}(t)^{3}+A r_{1}(t)\left(t^{2}+t+1\right)^{2}+b\left(t^{2}+t+1\right)^{3}\right)\left(t^{2}+t+1\right) .
$$

We define $E_{D} / \mathbb{Q}(t)$ by the equation

$$
D(t) y^{2}=x^{3}+A x+b,
$$

and put

$$
P_{i}=\left(\frac{r_{i}(t)}{t^{2}+t+1}, \frac{1}{\left(t^{2}+t+1\right)^{2}}\right) \text { for } i=1,2 .
$$

Here $P_{1}$ and $P_{2}$ are points from $E_{D}(\mathbb{Q}(t))$. The discriminant of $D$ is $3^{13} A^{12}\left(4 A^{3}+27 b^{2}\right)^{3}$ and so, by assumption, is non-zero. Let $E / \mathbb{Q}$ be given by $y^{2}=x^{3}+A x+b$. Let $C$ be the curve given by $s^{2}=D(t)$. Then, as in Proposition 1,

$$
\varphi_{P_{1}}^{*} \omega_{E}=-\frac{1}{2}\left((2 a+c) t^{2}+2(a+2 c) t-a+c\right) \frac{d t}{s}
$$

and

$$
\varphi_{P_{2}}^{*} \omega_{E}=-\frac{1}{2}\left((-a+c) t^{2}-2(2 a+c) t-a-2 c\right) \frac{d t}{s} .
$$


Since $a$ and $c$ are not both zero the differentials are non-zero and linearly independent. Thus, by Proposition 1 , the rank of $E_{D}(\mathbb{Q}(t))$ is at least 2. Our result now follows on appealing to Theorem 1 since the degree of $D$ is at most 8 and the largest irreducible factor of $D$ in $\mathbb{Q}[t]$ has degree at most 6.

Theorem 5. Let $a$ and $b$ be rational numbers with $a(3 a-b)(a+b) \neq 0$. There exist positive numbers $C_{50}$ and $C_{51}$, which depend on $a$ and $b$, such that if $T$ is a real number larger than $C_{50}$, then the number of square-free integers $d$ with $|d| \leq T$ for which the curve given by

$$
d y^{2}=a x^{3}+b x^{2}+b x+a
$$

has rank at least 2 is at least $C_{51} T^{\frac{1}{3}}$.

Proof. Let $D(t)=a t^{6}+b t^{4}+b t^{2}+a$ and denote by $E_{D} / \mathbb{Q}(t)$ the curve given by $D(t) y^{2}=a x^{3}+b x^{2}+b x+a$. The points $\left(t^{2}, 1\right)$ and $\left(t^{-2}, t^{-3}\right)$ from $E_{D}(\mathbb{Q}(t))$ are mapped by $\lambda$, as in Proposition 1 , to the differentials $t \frac{d t}{s}$ and $-\frac{d t}{s}$ respectively, on the curve $C$ given by $s^{2}=D(t)$. The discriminant of $D$ is $-64 a^{2}(3 a-b)^{6}(a+b)^{2}$ and our result now follows from Lemma 1 and Theorem 1 .

The last example of quadratic twists presented here yields twists of rank at least 3 for a class of elliptic curves. It is based on an idea of Schoen [36] and in fact was discovered independently by him.

One starts with an elliptic curve $E_{a}$ given by an equation

$$
E_{a}: y^{2}=x(x-1)(x-a) \text {. }
$$

We assume that both $a$ and $a-1$ are squares in $\mathbb{Q}^{*}$. Under this assumption the curves $E_{a}, E_{1 / a}$ and $E_{1 /(1-a)}$ are isomorphic over $\mathbb{Q}$. Note that the condition is equivalent to $a$ being of the form $\left(\frac{b^{2}+1}{2 b}\right)^{2}$ for $b \neq 0, \pm 1$ in $\mathbb{Q}$.

Using the projections $(x, y) \mapsto x: E \rightarrow \mathbb{P}^{1}$ one defines a fibre product

$$
C=E_{a} \times_{\mathbb{P}^{1}} E_{1 / a} \times_{\mathbb{P}^{1}} E_{1 /(1-a)} \text {. }
$$

Locally, $C$ can be described by the equations

$$
\left\{\begin{array}{l}
y_{1}^{2}=x(x-1)(x-a), \\
y_{2}^{2}=x(x-1)\left(x-\frac{1}{a}\right), \\
y_{3}^{2}=x(x-1)\left(x-\frac{1}{1-a}\right) .
\end{array}\right.
$$

One can describe $C$ alternatively using the functions $x, y_{1}$ and $u=\frac{y_{1} y_{2}}{x(x-1)}$, $v=\frac{y_{1} y_{3}}{x(x-1)}$. These satisfy the relations

$$
\left\{\begin{array}{l}
y_{1}^{2}=x(x-1)(x-a), \\
u^{2}=(x-a)\left(x-\frac{1}{a}\right), \\
v^{2}=(x-a)\left(x-\frac{1}{1-a}\right) .
\end{array}\right.
$$

It is easily checked that the latter two equations define a rational curve with a $\mathbb{Q}$-rational point. Parametrizing this curve allows us to prove our next result. 
Theorem 6. Let $b$ be a rational number with $b \neq 0,1,-1$. Put $a=\left(\frac{b^{2}+1}{2 b}\right)^{2}$. There exist positive numbers $C_{52}$ and $C_{53}$, which depend on $b$, such that if $T$ is a real number larger than $C_{52}$, then the number of square-free integers $d$ with $|d| \leq T$ for which the curve given by

$$
d y^{2}=x(x-1)(x-a)
$$

has rank at least 3 is at least $C_{53} T^{\frac{1}{6}}$.

Proof. Put $A=a^{3}-a^{2}-a+1, B=a^{3}-a^{2}+a$,

$$
U=\frac{A t^{2}-B}{A t^{2}-2 A t+B}, \quad V=\frac{A t^{2}-B}{-A t^{2}+2 B t-B} \quad \text { and } \quad x(t)=\frac{U^{2}-a^{2}}{a\left(U^{2}-1\right)} \text {. }
$$

Next put

$$
y(t)=\left(A t^{2}-B\right) /\left(8 a^{2}(a-1)^{2} t^{2}\left(A t^{2}-(A+B) t+B\right)^{2}\right)
$$

and

$$
D(t)=y(t)^{-2} x(t)(x(t)-1)(x(t)-a) .
$$

Let $E / \mathbb{Q}$ be defined by $y^{2}=x(x-1)(x-a)$ and $E_{D} / \mathbb{Q}(t)$ be defined by $D(t) y^{2}=x(x-1)(x-a)$. Notice that $P_{1}=(x(t), y(t)), P_{2}=\left(a x(t), a^{\frac{3}{2}} y(t) / U\right)$ and $P_{3}=\left(1+(a-1) x(t),(a-1)^{\frac{3}{2}} y(t) / V\right)$ are in $E_{D}(\mathbb{Q}(t))$. In the notation of Proposition 1,

$$
\begin{aligned}
& \varphi_{P_{1}}^{*} \omega_{E}=-a(a-1)\left(A t^{2}-2 B t+B\right)\left(A t^{2}-2 A t+B\right) \frac{d t}{s}, \\
& \varphi_{P_{2}}^{*} \omega_{E}=-a^{\frac{1}{2}}(a-1)\left(A t^{2}-2 B t+B\right)\left(A t^{2}-B\right) \frac{d t}{s} \\
& \varphi_{P_{3}}^{*} \omega_{E}=a(a-1)^{\frac{1}{2}}\left(A t^{2}-B\right)\left(A t^{2}-2 A t+B\right) \frac{d t}{s}
\end{aligned}
$$

and thus, since $A \neq B$, the rank of $E_{D}(\mathbb{Q}(t))$ is at least 3 .

The discriminant of $D$ is $2^{40} a^{70}(a-1)^{80}(a+1)^{20}\left(a^{2}-a+1\right)^{30}(2 a-1)^{30}$ and the degree of $D$ is 11 . Further $D$ factors as

$$
\begin{aligned}
-a & (a-1) t(t-1)(A t-B)\left(A t^{2}-\left(2 a^{3}-6 a^{2}+4 a\right) t+B\right) \\
& \cdot\left(A t^{2}-(A+B-1) t+B\right)\left((a-1)^{3} t^{2}-2\left(B-a^{2}\right) t+B\right) \\
& \cdot\left(\left(A+2 a^{2}-2\right) t^{2}-\left(2 a^{3}-2 a\right) t+B\right) .
\end{aligned}
$$

Thus we may apply Lemma 1 and, on clearing denominators in $D$, Theorem 1 to obtain our result.

\section{Cubic twists}

A cubic twist of an elliptic curve only exists if the elliptic curve admits an automorphism of degree 3 . This means that such a curve has $j$-invariant 0 , and it can be given by an equation $y^{2}=x^{3}+k$. 
Here we will restrict ourselves to the curves studied by Zagier and Kramarz [47] and Mai [28], which are described by the equation

$$
X^{3}+Y^{3}=m \text {. }
$$

Define a morphism of degree 3 by

$$
\xi=\frac{-X Y}{m}, \quad \eta=\frac{-Y^{3}}{m} .
$$

The image is the curve given by

$$
\eta^{2}+\eta=m \xi^{3}
$$

or equivalently in homogeneous coordinates by $\eta \zeta(\eta+\zeta)=m \xi^{3}$ which one can dehomogenize alternatively as

$$
x y(x+y)=m .
$$

The morphism of degree 3 does not affect the rank. The curves we look at are the cubic twists of the curve $E / \mathbb{Q}$ given by $x y(x+y)=1$. Note that Proposition 1 is applicable also for the curves given by our equation, since we can write it in the form $(8 m \eta+4 m)^{2}=(4 m \xi)^{3}+16 m^{2}$.

In a sense the most general parametrized twist $E_{t}$ of $E$ is given by the equation

$$
x y(x+y)=t \text {. }
$$

Since the curve defined by $s^{3}=t$ is rational, it follows from Proposition 1 that $\operatorname{rank} E_{t}(\mathbb{Q}(t))=0$. A special case of a conjecture of Silverman (see p. 556 of [41]) then claims that for almost all specializations, the curve $x y(x+y)=N$ (and hence also $X^{3}+Y^{3}=N$ ) has rank at most 1. This contrasts with the experimental data found by Zagier and Kramarz [47].

The next thing we want is a polynomial $m(t)$ such that the curve $C$ given by $s^{3}=m(t)$ admits many morphisms to $E$ with the property that the pullbacks of the standard invariant differential on $E$ are all in the same eigenspace for the action of the automorphism of order 3. One way to try to find such $m(t)$ is to look for cases where the zeroes of $m(t)$ in $\mathbb{P}^{1}$ admit a lot of symmetry with respect to the action of $\mathrm{PGL}_{2}(\mathbb{Q})$. This will give automorphisms on the curve $C$ and one can hope to find elliptic quotients to which the standard automorphism of degree 3 on $C$ descends, which will force such a quotient to have $j$-invariant 0 .

Such polynomials of degree 5 were studied by Igusa [21] who classified the genus 2 curves with additional automorphisms. One of them, suitably normalized for our purposes, is $m(t)$ where

$$
m(t)=2 t(t-1)(t+1)(2 t+1)(t+2) .
$$

Indeed our choice is motivated by some related work of Stewart [43] where the surface $x y(x+y)=m(t)$ is employed to prove that there are arbitrarily large integers $h$ for which the Thue equation $x y(z+y)=h$ has at least 18 solutions in coprime integers $x$ and $y$. 
We now take $C$ to be the curve given by $s^{3}=m(t)$. This gives a model of a genus 4 curve; a basis of the holomorphic differentials which will be convenient for us is given by

$$
\begin{gathered}
\omega_{1}=\frac{d t}{s}, \quad \omega_{2}=\left(1+t+t^{2}\right) \frac{d t}{s^{2}} \\
\omega_{3}=\left(1-2 t-2 t^{2}\right) \frac{d t}{s^{2}}, \quad \omega_{4}=\left(2+2 t-t^{2}\right) \frac{d t}{s^{2}} .
\end{gathered}
$$

On $C$ we define the involution

$$
\sigma_{1}(t, s)=\left(\frac{1-t}{1+2 t}, \frac{3 s}{(1+2 t)^{2}}\right) .
$$

One checks that the space of $\sigma_{1}^{*}$-invariant holomorphic differentials is generated by $\omega_{3}$. Hence the quotient of $C$ by $\sigma_{1}$ is elliptic; in fact invariant functions are

$$
x=\frac{t(t-1)}{s} \text { and } y=\frac{-(t+2)(t+1)}{s}
$$

which clearly satisfy the relation $x y(x+y)=1$.

A second involution is given by

$$
\sigma_{2}(t, s)=\left(\frac{t+2}{t-1}, \frac{3 s}{(t-1)^{2}}\right) .
$$

Here $\omega_{4}$ generates the space of invariant differentials. Invariant functions are given by

$$
u=\frac{(2 t+1)(t+1)}{s} \text { and } v=\frac{t-1}{s}
$$

which also satisfy $u v(u+v)=1$.

One can find another involution defined as

$$
\sigma_{3}(t, s)=\left(-\frac{t+2}{2 t+1}, \frac{-3 s}{(2 t+1)^{2}}\right) .
$$

This one has $\omega_{2}$ as invariant differential and the functions

$$
w=2 \frac{2 t+1}{s} \text { and } z=2 \frac{t^{2}-1}{s}
$$

are invariant. They satisfy $w z(w+z)=4$. This equation defines an elliptic curve which has bad reduction at 2 while the curve given by $x y(x+y)=1$ has good reduction at 2 . Hence over $\mathbb{Q}$ the two elliptic curves are not isogenous.

Since this exhausts the biggest eigenspace for the action of the automorphism of degree 3, recall the proof of Corollary 1 , we conclude that the $\mathbb{Q}(t)$-rank of the elliptic curve given by $x y(x+y)=m(t)$ is 2 . From a geometric point of view it might be interesting to note the following result.

Proposition 2. The smooth minimal surface associated with the equation

$$
x y(x+y)=2 t(t-1)(t+1)(2 t+1)(t+2)
$$


is an elliptic K3-surface with Picard number 20. As an elliptic surface over the t-line it has rank 6.

Proof. The fact that this defines a K3-surface is easily verified using the criterion given in [1], pp. 276-277. Using Tate's algorithm [44], pp. 46-52, one finds that this surface has six bad fibers. Each of these is of type IV which means that they look like three rational curves meeting transversally in one point.

The Shioda-Tate formula for the Picard number $\rho$ then says that

$$
\rho=r+2+6 \cdot 2=r+14,
$$

with $r$ the $\mathbb{C}(t)$-rank of the elliptic curve defined by our equation. The discussion preceding this proposition implies that the $\mathbb{Q}(\sqrt[3]{4}, t)$-rank is at least 3 . Hence using the action of the endomorphism ring on three such independent points one obtains a $\mathbb{Z}$-module of rank 6 . It follows that $\rho \geq 6+14=20$. Since for all K3-surfaces the inequality $\rho \leq 20$ holds, this proves the proposition.

We will now discuss rank 3 twists of $X^{3}+Y^{3}=1$. Write

$$
m(t)=2 A(t) B(t) C(t) D(t) F(t) G(t)
$$

with $A(t)=t, B(t)=1, C(t)=t-1, D(t)=t+1, F(t)=t+2$ and $G(t)=2 t+1$. Our aim is to find for many $t$ 's a point $(a, b)$ on $x y(x+y)=$ $2 A B C D F G$ which is independent from the two points we already have.

Our basic idea is to try $(a, b)$ such that $a+b=\lambda A B$. This choice of two of the six forms $A, \ldots, G$ may seem arbitrary, but in fact it is not. The points we had before also correspond to such pairs. Using isomorphisms of $C$ one can change from one pair to another; on $E$ this corresponds to such things as translation by a point of order 3 or the [-1]-map. In terms of such transformations, the pair $(A, B)$ is the only one we have not yet discussed.

If a solution $(a, b)$ satisfies $a+b=\lambda A B$, then obviously $a, b$ are roots of the equation $X^{2}-\lambda A B X+2 C D F G / \lambda=0$. Hence the discriminant of this expression, or equivalently

$$
\lambda^{2} t^{2}-\frac{16}{\lambda} t^{4}-\frac{40}{\lambda} t^{3}+\frac{40}{\lambda} t+\frac{16}{\lambda},
$$

has to be a square. This is described by a family of elliptic curves over the $\lambda$-line; for example sections are given by $t= \pm 1$.

Originally we looked for fibers in this family which have infinitely many $\mathbb{Q}$ rational points. Then base changing the family given by $x y(x+y)=m(t)$ over the $t$-line to such an elliptic curve yields a family with three independent sections; the third one given by the point $(a, b)$. Specializing to rational points on that elliptic curve then yields, in general, twists $x y(x+y)=m$ with rank at least 3. The main disadvantage of this method is that one obtains at best very weak density results on the number of twists obtained in this way. The point is that the number of rational points on an elliptic curve which have bounded height is much smaller than the number of such points on a rational curve.

One resolves this problem by taking instead of a base change to a fiber, a base change to a section of the family

$$
Y^{2}=\lambda^{2} t^{2}-\frac{16}{\lambda} t^{4}-\frac{40}{\lambda} t^{3}+\frac{40}{\lambda} t+\frac{16}{\lambda} .
$$


Taking the point $(t=1, Y=\lambda)$ as zero element for the group law, we computed that twice the point $(t=-1, Y=\lambda)$, which itself only leads to a degenerate 'twist' $X^{3}+Y^{3}=0$, equals the point

$$
\left(t=\frac{1-\lambda^{3}}{1+\lambda^{3}}, Y=\frac{\lambda\left(\lambda^{6}-17\right)}{\left(1+\lambda^{3}\right)^{2}}\right) .
$$

The base change of the family $x y(x+y)=m(t)$ to this section can be seen as a family over the $\lambda$-line, written as

$$
x y(x+y)=m\left(\frac{1-\lambda^{3}}{1+\lambda^{3}}\right)
$$

As an elliptic curve over $\mathbb{Q}(\lambda)$ this is isomorphic to

$$
x y(x+y)=\left(1-\lambda^{3}\right)\left(1+\lambda^{3}\right)\left(3+\lambda^{3}\right)\left(3-\lambda^{3}\right) .
$$

On this model the point $(a, b)$ which we have constructed can be written as

$$
\left(4, \frac{\lambda^{6}-9}{2}\right)
$$

To check that the three points we now have are indeed independent one studies the corresponding differentials. Our points yield morphisms from the curve given by $s^{3}=\left(\lambda^{6}-1\right)\left(\lambda^{6}-9\right)$ to the elliptic curve given by $x y(x+y)=1$. An invariant differential on this elliptic curve can be written as $\frac{d x}{x(2 y+x)}$. The third point we constructed corresponds to the morphism

$$
(\lambda, s) \mapsto\left(x=\frac{4}{s}, y=\frac{\lambda^{6}-9}{2 s}\right) .
$$

From this description it is clear that the pull back of any differential on $E$ is in the +1 -eigenspace of the action on differentials of the automorphism given by $(\lambda, s) \mapsto\left(\zeta_{6} \lambda, s\right)$. The other two differentials are pullbacks under the morphism given by

$$
\left(\lambda, s^{\prime}\right) \mapsto\left(t=\frac{1-\lambda^{3}}{1+\lambda^{3}}, s=\frac{-2 \lambda s^{\prime}}{\left(1+\lambda^{3}\right)^{2}}\right)
$$

to the curve given by $s^{3}=2 t(t-1)(t+1)(t+2)(2 t+1)$. It follows easily that they are not in the +1 -eigenspace of the automorphism mentioned.

Thus the elliptic curve defined over the function field $\mathbb{Q}(\lambda)$ by the equation

$$
x y(x+y)=\left(\lambda^{6}-1\right)\left(\lambda^{6}-9\right)
$$

has $\mathbb{Q}(\lambda)$-rank at least 3. We remark that we are able to prove that the $\mathbb{Q}(\lambda)$-rank is 3 and that the $\mathbb{C}(\lambda)$-rank is 14 ; we do not give the details here.

We shall now apply the above results to estimate the number of cubic twists of the elliptic curve given by $x^{3}+y^{3}=1$ which have rank at least 2 or rank at least 3 . 
Theorem 7. There exist positive numbers $C_{54}, C_{55}$, and $C_{56}$ such that if $T$ is a real number larger than $C_{54}$, then the number of cube-free integers $d$ with $|d| \leq T$ for which the curve given by

$$
x^{3}+y^{3}=d
$$

has rank at least 2 is at least $C_{55} T^{\frac{1}{3}}$, and for which it has rank at least 3 is at least $C_{56} T^{\frac{1}{6}}$.

Proof. This result follows from Lemma 1, our discussion above and Theorem 1. For the case of rank 2 we apply Theorem 1 to the binary form $F(X, Y)=$ $2 X Y(X-Y)(X+Y)(2 X+Y)(X+2 Y)$ whereas for the case of rank 3 we take $F(X, Y)=\left(X^{6}-Y^{6}\right)\left(X^{6}-9 Y^{6}\right)$.

\section{QUARTIC TWISTS}

The only elliptic curves over $\mathbb{Q}$ for which quartic twists exist are those with $j$-invariant 1728. Such curves can be given by an equation $y^{2}=x^{3}+a x$.

Let $E / \mathbb{Q}$ be the elliptic curve given by the equation $y^{2}=x^{3}+x$. To get quartic twists of $E$ of rank 2 we take $D(t)=-t^{4}-1$ and let $E_{D} / \mathbb{Q}(t)$ be given by the equation $y^{2}=x^{3}+D(t) x$. Notice that $P_{1}=\left(-t^{2}, t\right)$ and $P_{2}=\left(-1, t^{2}\right)$ are in $E_{D}(\mathbb{Q}(t))$. Further on taking $C$ to be the Fermat curve $s^{4}=-t^{4}-1$ and following the notation of Proposition 1 we find that $\varphi_{P_{1}}^{*} \omega_{E}=\frac{d t}{s^{3}}$ and $\varphi_{P_{2}}^{*} \omega_{E}=-t \frac{d t}{s^{3}}$. Thus the rank of $E_{D}(\mathbb{Q}(t))$ is at least 2 .

Mestre [30] has shown how to find polynomials $D$ for which the rank of $E_{D}(\mathbb{Q}(t))$ is at least 4 . He proceeds as follows. Let $x_{1}, x_{2}, x_{3}$ be in $\mathbb{Q}(t)$. Put $x_{4}=-\left(x_{1}+x_{2}+x_{3}\right), p(x)=\left(x-x_{1}\right)\left(x-x_{2}\right)\left(x-x_{3}\right)\left(x-x_{4}\right)=x^{4}+a_{2} x^{2}+a_{1} x+a_{0}$ and $P_{i}=\left(x_{i}, x_{i}\right)$ for $i=1,2,3,4$. Plainly $P_{i}$ is on the curve given by $x^{4}+a_{2} y^{2}+a_{1} y+a_{0}=0$ for $i=1,2,3,4$. Further, if $a_{0}=-u^{4}$ with $u$ in $\mathbb{Q}(t)$, then $(u, 0)$ is also on the curve. Note that if $X=-4 a_{2} x^{2}$ and $Y=4 a_{2} x\left(2 a_{2} y+a_{1}\right)$, then $Y^{2}=X^{3}+4 a_{2}\left(4 a_{2} a_{0}-a_{1}^{2}\right) X$.

Mestre [30] takes $u=1$ and, appealing to a parametric solution due to Euler of $a_{0}=-1$, he chooses

$$
x_{1}=t\left(\frac{2 t^{2}-1}{2 t^{2}+1}\right), \quad x_{2}=\frac{\left(2 t^{2}-1\right)}{2 t\left(2 t^{2}+1\right)} \quad \text { and } \quad x_{3}=\frac{4 t}{2 t^{2}-1} .
$$

Mestre then proves that the curve he gets when he specializes to $t=1$ has rank at least 4 and so $E_{D_{0}}(\mathbb{Q}(t))$ has rank at least 4 where $D_{0}(t)=4 a_{2}\left(4 a_{2} a_{0}-a_{1}^{2}\right)$. Using the above ideas we shall prove our next result.

Theorem 8. There exist positive numbers $C_{57}, C_{58}, C_{59}$ and $C_{60}$ such that if $T$ is a real number larger than $C_{57}$, then the number of fourth power free integers $d$ with $|d| \leq T$ for which the curve given by

$$
y^{2}=x^{3}+d x
$$


has rank at least 2,3 or 4 is at least $C_{58} T^{\frac{1}{2}}, C_{59} T^{\frac{1}{4}}$ or $C_{60} T^{\frac{1}{16}} /(\log T)^{2}$ respectively.

Proof. By the above discussion the rank of $E_{D}(\mathbb{Q}(t))$ is at least 2 when $D(t)=$ $-t^{4}-1$. Applying Lemma 1 and Theorem 1 the result for the case of rank 2 follows.

Define $D_{0}(t)$ as above and put $D_{1}(t)=\left(2 t\left(2 t^{2}-1\right)\left(2 t^{2}+1\right)^{2}\right)^{4} D_{0}(t)$. Then

$$
\begin{aligned}
D_{1}(t)= & \left(2 t^{2}+3\right)\left(6 t^{2}+1\right)\left(4 t^{4}+12 t^{2}+1\right)\left(12 t^{4}+4 t^{2}+3\right) \\
& \cdot\left(64 t^{12}+32 t^{10}+304 t^{8}+176 t^{6}+76 t^{4}+2 t^{2}+1\right)\left(2 t^{2}+1\right)^{2}\left(2 t^{2}-1\right)^{2} .
\end{aligned}
$$

Note that the rank of $E_{D_{1}}(\mathbb{Q}(t))$ is at least 4 since it equals the rank of $E_{D_{0}}(\mathbb{Q}(t))$ which is at least 4. Put $D_{2}(t)=D_{1}(t) /\left(\left(2 t^{2}+1\right)\left(2 t^{2}-1\right)\right)$. Since the degree of $D_{1}$ is 32 and the discriminant of $D_{2}$ is $-2^{854} 3^{12} 5^{16} 83^{4}$ we may apply Lemma 1 and Theorem 2 to deduce our result for the case of rank 4 .

If we choose $x_{1}=1, x_{2}=t$ and $x_{3}=t+2$ in Mestre's construction and put $D(t)=16\left(t^{2}-3\right)\left(3 t^{2}+8 t+7\right)\left(t^{2}+t-1\right)\left(t^{2}+3 t+3\right)$, then we deduce from Proposition 1 in the usual manner that the rank of $E_{D}(\mathbb{Q}(t))$ is at least 3 where $E_{D}$ is given by $y^{2}=x^{3}+D(t) x$. Since the discriminant of $D$ is $2^{76} 3^{6} 5^{6}$ and the degree of $D$ is 8 our result follows from Lemma 1 and Theorem 1.

\section{SeXtic Twists}

The only elliptic curves over $\mathbb{Q}$ for which sextic twists exist are those with $j$-invariant 0 . Such curves can be given by an equation $y^{2}=x^{3}+k$.

Let $E / \mathbb{Q}$ be the elliptic curve given by the equation $y^{2}=x^{3}+1$. We shall employ two different constructions to produce twists of $E$ of large rank. The first construction produces twists of $E$ of rank 3 and 4 while the second construction, due to Mestre [30], produces twists of rank 5 and 6 .

We shall now describe our construction of rank 3 and rank 4 twists. Let $x_{1}, x_{2}$ and $x_{3}$ be in $\mathbb{Q}(t)$ and put $F(X, Y)=\left(X-x_{1} Y\right)\left(X-x_{2} Y\right)\left(X-x_{3} Y\right)+$ $Y^{3}$. Put $P_{i}^{\prime}=\left(x_{i}, 1\right)$ for $i=1,2,3$ and $P_{4}^{\prime}=(1,0)$. Plainly $P_{1}^{\prime}, P_{2}^{\prime}, P_{3}^{\prime}$ and $P_{4}^{\prime}$ are points on the curve $E_{F}$ given by $F(X, Y)=1$. We shall choose $x_{1}, x_{2}$ and $x_{3}$ so that the rank of the $\mathbb{Q}(t)$-points of $E_{F}$ is at least 3. If, in addition, $-x_{1} x_{2} x_{3}+1$ is a cube in $\mathbb{Q}(t)$, say $v^{3}$, then $P_{5}^{\prime}=\left(0, \frac{1}{v}\right)$ is another point on $F(X, Y)=1$ and an appropriate choice of $x_{1}, x_{2}$ and $x_{3}$ will ensure that the rank of $E_{F}(\mathbb{Q}(t))$ is at least 4. Let $H(X, Y)$ be the quadratic covariant of $F$, let $G(X, Y)$ be the cubic covariant of $F$ and let $D_{0}$ be the discriminant of $F$. We have

$$
(4 G)^{2}=(4 H)^{3}-27 \cdot 16 \cdot D_{0} \cdot F^{2}
$$

(see Chapter 24 of [32]), and so there is a morphism defined over $\mathbb{Q}(t)$ from $E_{F}$ to the curve $E_{D}$ given by $y^{2}=x^{3}+D$ where $D=-27 \cdot 16 \cdot D_{0}$. Thus we obtain a family of sextic twists of $E$.

Our initial construction of families of rank 5 and rank 6 twists of $E$ depended on work of Craig [8], [9]. In [8] Craig proved that infinitely many 
imaginary quadratic fields have a subgroup of the class group isomorphic to the direct product of three copies of the cyclic group of order 3 . To do so he constructed a polynomial $D$ of degree 24 (see $\S 4$ of [8]) and exhibited five different points in $E_{D}(\mathbb{Q}(t))$. Using Proposition 1 one may check that the rank of $E_{D}(\mathbb{Q}(t))$ is at least 5. Since the discriminant of $D$ is $-2^{184} 3^{300} 7^{18} 647^{3}$ we may apply Theorem 2 to deduce that rank 5 twists of $E$ occur with exponent $\frac{1}{12}$. In [9] Craig sharpened his earlier result by proving that there are infinitely many imaginary quadratic fields for which the 3-rank of the ideal class group is at least 4. Nakano [33] deduced from Craig's construction that there are infinitely many twists of $E$ of rank 6. Using the polynomial $D$ of $\S 8$ of [9] and proceeding in the usual manner one finds that rank 6 twists of $E$ occur with exponent $\frac{1}{72}$.

We are able to obtain larger exponents however, by employing the following construction of Mestre [30]. Let $x_{1}, \ldots, x_{5}$ be in $\mathbb{Q}(t)$. Put

$$
\begin{aligned}
x_{6} & =-\left(x_{1}+\cdots+x_{5}\right), \\
p(X) & =\left(X-x_{1}\right) \cdots\left(X-x_{6}\right) \\
& =X^{6}+a_{4} X^{4}+a_{3} X^{3}+a_{2} X^{2}+a_{1} X+a_{0}, \\
g(X) & =X^{2}+a_{4} / 3
\end{aligned}
$$

and

$$
r(X)=r_{3} X^{3}+r_{2} X^{2}+r_{1} X+r_{0},
$$

where $r_{3}=a_{3}, r_{2}=a_{2}-a_{4}^{2} / 3, r_{1}=a_{1}$ and $r_{0}=a_{0}-\left(a_{4} / 3\right)^{3}$. Next put

$$
F(X, Y)=r_{3} X^{3}+r_{2} X^{2} Y+r_{1} X Y^{2}+r_{0} Y^{3},
$$

let $E_{F}$ denote the curve $F(X, Y)=-1$ and put $P_{i}^{\prime}=\left(\frac{x_{i}}{g\left(x_{i}\right)}, \frac{1}{g\left(x_{i}\right)}\right)$ for $i=$ $1, \ldots, 6$. We shall choose $x_{1}, \ldots, x_{5}$ so that $E_{F}(\mathbb{Q}(t))$ has rank at least 5 . Further if $r_{3}$ is a cube in $\mathbb{Q}(t)$, say $v^{3}$, then $P_{7}^{\prime}=\left(-\frac{1}{v}, 0\right)$ is in $E_{F}(\mathbb{Q}(t))$. Mestre takes

$$
\begin{aligned}
x_{1}=-x_{2} & =126(35 t-19)(14 t-13)(t+1), \\
x_{3} & =63\left(-980 t^{3}+3549 t^{2}-3084 t+1135\right), \\
x_{4} & =63\left(1127 t^{3}-3108 t^{2}+3525 t-988\right), \\
x_{5} & =-113876 t^{3}+265629 t^{2}-259980 t+69103
\end{aligned}
$$

and

$$
x_{6}=104615 t^{3}-293412 t^{2}+232197 t-78364 .
$$

In this case $r_{3}=\left(2 x_{1} / 3\right)^{3}$ and by specializing to $t=1$ he proves that the rank of $E_{F}(\mathbb{Q}(t))$ is at least 6. We now use (23) as before to obtain sextic twists of $E$. 
Theorem 9. There exist positive numbers $C_{61}, C_{62}, C_{63}, C_{64}$ and $C_{65}$ such that if $T$ is a real number larger than $C_{61}$, then the number of sixth power free integers $d$ with $|d| \leq T$ for which the curve given by

$$
y^{2}=x^{3}+d
$$

has rank at least $3,4,5$ or 6 is at least $C_{62} T^{\frac{1}{3}}, C_{63} T^{\frac{1}{6}}, C_{64} T^{\frac{1}{9}} /(\log T)^{2}$ or $C_{65} T^{\frac{1}{27}} /(\log T)^{2}$ respectively.

Proof. We shall prove the result for rank 3 and 4 twists with the first construction we discussed above. Accordingly take $x_{1}=1, x_{2}=t, x_{3}=2 t+1$. Then using (23) and Proposition 1 we find that the rank of $E_{D}(\mathbb{Q}(t))$ is at least 3 where $E_{D}$ is given by $y^{2}=x^{3}+D(t)$ and

$$
D(t)=2^{4} 3^{3}\left(-4 t^{6}+8 t^{4}-40 t^{2}+31\right) \text {. }
$$

Our result now follows from Lemma 1 and Theorem 1 on noting that the discriminant of $D$ is $2^{56} 3^{30} 5^{6} 7^{6} 31$.

For rank 4 twists we take $x_{1}=\left(1-t^{2}\right) / 2, x_{2}=2\left(t^{2}+t+1\right)$ and $x_{3}=t^{2}-t+1$. Then, using (23) and Proposition 1 we find that the rank of $E_{D}(\mathbb{Q}(t))$ is at least 4 where $E_{D}$ is given by $y^{2}=x^{3}+D(t)$ and

$$
\begin{aligned}
D(t)= & -6075 t^{12}-38070 t^{11}-81513 t^{10}-83106 t^{9}-67797 t^{8}-39528 t^{7} \\
& -27270 t^{6}-58968 t^{5}-89181 t^{4}-84834 t^{3}-52353 t^{3}-23814 t+9261 .
\end{aligned}
$$

As before, our result follows from Lemma 1 and Theorem 1 on noting that the discriminant of $D$ is $2^{64} 3^{72} 5^{6} 7^{18} 13^{3} 31^{3} 43^{3} 73^{3} 241^{3} 13807861$.

For rank 5 twists we take $x_{1}=1, x_{2}=2, x_{3}=-3, x_{4}=0, x_{5}=t$ and $x_{6}=-t$ in Mestre's construction. Again by (23) and Proposition 1 we find that the rank of $E_{D}(\mathbb{Q}(t))$ is at least 5 where $E_{D}$ is given by $y^{2}=x^{3}+D(t)$ and

$$
D(t)=\frac{-64}{27}\left(t^{18}+2973 t^{12}-369249 t^{6}+11764900\right) \text {. }
$$

Our result now follows from Lemma 1 and Theorem 2 on noting that the discriminant of $D$ is $-2^{232} 3^{30} 5^{28} 7^{48} 11^{18}$.

Finally with the choice of $x_{1}, \ldots, x_{6}$ made by Mestre above and the transformation (23) we obtain $D(t)$ for which the rank of $E_{D}(\mathbb{Q}(t))$ is at least 6 . The degree of $D$ is 54 and the discriminant

$$
2^{2756} 3^{8838} 5^{18} 7^{2886} 13^{24} 17^{6} 19^{18} 23^{3} 31^{16} 47^{6} \ldots
$$

is non-zero. Our result follows from Lemma 1 and Theorem 2.

\section{PARITY}

In this section we briefly recall a conjecture on the parity of the rank of the Mordell-Weil group of an elliptic curve. We shall restrict our attention to the case of twists of a given curve.

Let $E / \mathbb{Q}$ be an elliptic curve with conductor $N$. Suppose that $E$ is given by the equation $y^{2}=f(x)$. Let $d$ be a square-free non-zero integer and $E_{d}$ be given by the equation $d y^{2}=f(x)$. Let $r(d)$ denote the rank of $E_{d}(\mathbb{Q})$. 
Parity conjecture for quadratic twists. Suppose that $d$ and $2 N$ are coprime. Then

$$
(-1)^{r(d)-r(1)}=\chi_{d}(-N),
$$

where $\chi_{d}$ is the quadratic Dirichlet character belonging to the field $\mathbb{Q}(\sqrt{d})$.

We refer to $\S 2$ of [5] for a discussion of this conjecture; see [22], [23] for some recent work on this problem. We also have corresponding conjectures for cubic, quartic and sextic twists. Birch and Stephens [2] worked out explicit versions of these conjectures in the cubic and quartic cases. Recently Liverance [25] has done this for the sextic case.

Parity conjecture for cubic twists. Let $d$ be a cube free integer and let $E_{d}$ be the elliptic curve given by $x^{3}+y^{3}=d$. Let $r(d)$ denote the rank of $E_{d}(\mathbb{Q})$. We have

$$
(-1)^{r(d)}=-w_{3} \cdot \prod_{p \neq 3} w_{p}
$$

where

$$
w_{3}= \begin{cases}-1 & \text { if } d \equiv \pm 1, \pm 3(\bmod 9) \\ 1 & \text { otherwise }\end{cases}
$$

and

$$
w_{p}= \begin{cases}-1 & \text { if } p \mid d \text { and } p \equiv 2(\bmod 3) \\ 1 & \text { otherwise. }\end{cases}
$$

Parity conjecture for quartic twists. Let $d$ be a fourth power free integer and suppose $d \not \equiv 0(\bmod 4)$. Let $E_{d}$ be the curve given by $y^{2}=x^{3}+d x$ and let $r(d)$ denote the rank of $E_{d}(Q)$. Then

$$
(-1)^{r(d)}=\frac{d}{|d|} \cdot w_{2} \cdot \prod_{p \neq 2} w_{p}
$$

where

$$
w_{2}= \begin{cases}-1 & \text { if } d \equiv 1,3,11,13(\bmod 16) \\ 1 & \text { otherwise }\end{cases}
$$

and

$$
w_{p}= \begin{cases}-1 & \text { if } p^{2} \| d \text { and } p \equiv 3(\bmod 4) \\ 1 & \text { otherwise. }\end{cases}
$$

Note that the condition $d \not \equiv 0(\bmod 4)$ above is not significant since $r(d)=$ $r(-4 d)$. This follows from the fact that the curves $E_{d}$ and $E_{-4 d}$ are 2isogenous over $\mathbb{Q}$.

Parity conjecture for sextic twists. Let $d$ be a sixth power free integer with $d$ factoring as $d=2^{u} 3^{v} d_{6}$ where $\left(d_{6}, 6\right)=1$. Let $E_{d}$ be the curve given by $y^{2}=x^{3}+d$ and let $r(d)$ denote the rank of $E_{d}(\mathbb{Q})$. Then

$$
(-1)^{r(d)}=-w_{2} w_{3} \prod_{p \neq 2,3} w_{p}
$$


where

$$
\begin{aligned}
& w_{2}= \begin{cases}-1 & \text { if } 2 \nmid u \text { or } 2 \mid u, d_{2} \equiv 1(\bmod 4), u \neq 4 \\
+1 & \text { if } 2 \mid u, d_{2} \equiv-1(\bmod 4) \text { or } u=4, d_{2} \equiv 1(\bmod 4),\end{cases} \\
& w_{3}= \begin{cases}-1 & \text { if } v \equiv-1(\bmod 3) \text { or } 3 \mid v, d_{3} \equiv \pm 2(\bmod 9) \\
+1 & \text { if } v \equiv+1(\bmod 3) \text { or } 3 \mid v, d_{3} \equiv \pm 4(\bmod 9) \\
(-1)^{v}\left(\frac{d_{3}}{3}\right) \text { if } 3 \mid v, d_{3} \equiv \pm 1(\bmod 9)\end{cases} \\
& w_{p}= \begin{cases}-1 & \text { if } p \mid d, p \equiv 2(\bmod 3) \\
+1 & \text { otherwise }\end{cases}
\end{aligned}
$$

and where $d_{n}$ is the largest divisor of $d$ prime to $n$ (with the same sign as $d$ ), so that $d_{2}=3^{v} d_{6}$ and $d_{3}=2^{u} d_{6}$.

In many instances it is possible to employ the above conjectures to conclude that, for reasons of parity, the rank of a twist of a given elliptic curve is larger by one than our constructions indicate. This is the approach taken by Gouvêa and Mazur [15]. Let $E / \mathbb{Q}$ be given by the equation $y^{2}=x^{3}+A x+B$. They observe that if $D(t)=t^{3}+A t+B$, then certainly the point $(t, 1)$ is in $E_{D}(\mathbb{Q}(t))$ where $E_{D}$ is given by $D(t) y^{2}=x^{3}+A x+B$. Let $C$ be the conductor of $E$ and observe that we may assume that $A$ and $B$ are integers divisible by $M$ where $M=12 \cdot C$ by changing the model for $E$. By the parity conjecture for quadratic twists and the law of quadratic reciprocity there is a set $U$, consisting of half of the coprime residue classes modulo $M$, with the property that if $d$ is positive and belongs to a member of $U$, then the rank of $E_{d}$ is even. Put $F(X, Y)=Y\left(X^{3}+A X Y^{2}+B Y^{3}\right)$ and $F_{1}(X, Y)=F(X+j M Y, Y)$ where $j$ is the smallest positive integer for which $F_{1}(X, 1)$ has non-positive roots. Since $F_{1}(X, Y) \equiv Y X^{3}(\bmod M)$ there are integers $a_{0}$ and $b_{0}$ for which $F_{1}\left(a_{0}, b_{0}\right)$ belongs to a member of $U$. Thus, as noted by Gouvêa and Mazur, whenever $d=F_{1}(a, b)$ is sufficiently large, with $a \equiv a_{0}(\bmod M)$ and $b \equiv b_{0}(\bmod M)$, the rank of $E_{d}$ is at least 1 and so, by the parity conjecture, is even and at least 2. In our proof of Theorem 1 we estimate $R_{k}(x)$ by considering terms $F(a, b)$ where $a$ and $b$ are positive integers. Since $F_{1}(a, b)$ is positive when $a$ and $b$ are positive, we may appeal to Theorem 1 to obtain a slight refinement of their result; the exponent of $\frac{1}{2}-\varepsilon$ in their lower bound may be replaced by $\frac{1}{2}$.

Similarly we may recover the result of Mai on cubic twists of $E$ where $E / \mathbb{Q}$ is given by the equation $X^{3}+Y^{3}=1$. Let $D(t)=t^{3}+1$ and observe that $(t, 1)$ is a point in $E_{D}(\mathbb{Q}(t))$ where $E_{D}$ is given by $X^{3}+Y^{3}=D(t)$. On recalling the discussion at the start of $\S 9$ and applying Proposition 1 we see that the rank of $E_{D}(\mathbb{Q}(t))$ is at least 1. Put $F(X, Y)=X^{3}+Y^{3}$ and note that if $X \equiv 1(\bmod 9)$ and $Y \equiv 0(\bmod 9)$, then $X^{3}+Y^{3} \equiv 1(\bmod 9)$. If $d$ is a square-free positive integer with $d \equiv 1(\bmod 9)$, then the number of primes congruent to 2 modulo 3 which divide $d$ is even. Thus, by the parity conjecture for cubic twists, the rank of $X^{3}+Y^{3}=d$ is even.

Observe that $F(a, b)$ is positive when $a$ and $b$ are positive. Thus, by Theorem 1, there are positive numbers $C_{66}$ and $C_{67}$ such that the number of square-free positive integers $t$ with $t \leq x$ for which there are integers $a$ and 
$b$ with $a \equiv 1(\bmod 9), b \equiv 0(\bmod 9)$ and $a^{3}+b^{3}=t$ is at least $C_{66} x^{2 / 3}$ for $x$ at least $C_{67}$. Therefore, by Lemma 1 and the parity conjecture for cubic twists, there are positive numbers $C_{68}$ and $C_{69}$ such that the number of positive integers $d$ with $d \leq x$ for which the rank of $X^{3}+Y^{3}=d$ is even and at least 2 is at least $C_{68} x^{2 / 3}$ for $x$ greater than $C_{69}$.

As a final application let $F(X, Y)=\left(X^{6}-Y^{6}\right)\left(X^{6}-9 Y^{6}\right)$ and observe that if $a$ and $b$ are positive integers with $a<b$, then $F(a, b)$ is positive. It follows, as in the proof of Lemma 2, that the number of pairs of integers $(a, b)$ with $1 \leq a \leq x, 1 \leq b \leq x, a<b, a \equiv 1(\bmod 9)$ and $b \equiv 0(\bmod 9)$ for which $F(a, b)$ is square-free is $\frac{1}{2} C_{13} x^{2}+O\left(x^{2} /(\log x)^{\frac{1}{3}}\right)$; the estimates for the error terms in the proof are unchanged. Similarly, on modifying the proof of Theorem 1 by replacing $U$ by the set of pairs of integers $(a, b)$ in $U$ with $a<b$ and using the above observation we deduce that there are positive numbers $C_{70}$ and $C_{71}$ such that if $x$ exceeds $C_{70}$, then the number of square-free positive integers $t$ with $t \leq x$ and $t \equiv 1(\bmod 9)$ for which there exist integers $a$ and $b$ with $F(a, b)=t$ is at least $C_{71} x^{\frac{1}{6}}$. Thus by Lemma 1 , Proposition 3 and the parity conjecture for cubic twists there exist positive numbers $C_{72}$ and $C_{73}$ such that the number of positive integers $d$ with $d \leq x$ for which the rank of $X^{3}+Y^{3}=d$ is even and at least 4 is at least $C_{72} x^{\frac{1}{6}}$ for $x$ greater than $C_{73}$.

\section{REFERENCES}

1. F. Beukers and J. Stienstra, On the Picard-Fuchs equation and the formal Brauer group of certain elliptic K3-surfaces, Math. Ann. 271 (1985), 269-304.

2. B. J. Birch and N. Stephens, The parity of the rank of the Mordell-Weil group, Topology 5 (1966), 295-299.

3. B. J. Birch and H. P. F. Swinnerton-Dyer, Notes on elliptic curves. I, J. Reine Angew. Math. 212 (1963), 7-25.

4. __ Notes on elliptic curves. II, J. Reine Angew. Math. 218 (1965), 79-108.

5. __ Elliptic curves and modular functions, Modular Functions of One Variable IV (B. J. Birch and W. Kuyk, eds.), Lecture Notes in Math., vol. 476, Springer-Verlag, Berlin, New York, and Heidelberg, 1975, pp. 2-32.

6. A. Brumer, The average rank of elliptic curves. I, Invent. Math. 109 (1992), 445-472.

7. A. Brumer and O. McGuinness, The behaviour of the Mordell-Weil group of elliptic curves, Bull. Amer. Math. Soc. (N.S.) 23 (1990), 375-382.

8. M. Craig, A type of class group for imaginary quadratic fields, Acta Arith. 22 (1973), 449-459.

9. ___ A construction for irregular discriminants, Osaka J. Math. 14 (1977), 365-402.

10. D. S. Dummit, Néron models, Tate curves and Mestre's technique of using elliptic curves to construct quadratic fields with non-trivial 5-rank, Number Theory Seminar, Montréal, 1989.

11. P. Erdös and K. Mahler, On the number of integers which can be represented by a binary form, J. London Math. Soc. 13 (1938), 134-139.

12. G. Frey, On the Selmer group of twists of elliptic curves with $\mathbb{Q}$-rational torsion points, Canad. J. Math. 40 (1988), 649-665.

13. M. Fried, Constructions arising from Néron's high rank curves, Trans. Amer. Math. Soc. 281 (1984), 615-631. 
14. D. Goldfeld, Conjectures on elliptic curves over quadratic fields, Number Theory (Proc. Conf. in Carbondale, 1979) (M. B. Nathanson, ed.), Lecture Notes in Math., vol. 751, Springer-Verlag, Berlin, New York, and Heidelberg, 1979, pp. 108-118.

15. F. Gouvêa and B. Mazur, The square-free sieve and the rank of elliptic curves, J. Amer. Math. Soc. 4 (1991), 1-23.

16. G. Greaves, Power-free values of binary forms, Quart. J. Math. Oxford Ser. (2) 43 (1992), 45-65.

17. G. H. Hardy and E. M. Wright, An introduction to the theory of numbers, 5th ed., Oxford Univ. Press, London and New York, 1979.

18. T. Honda, Isogenies, rational points and section points of group varieties, Japan. J. Math. 30 (1960), 84-101.

19. C. Hooley, On power-free values of polynomials, Mathematika 14 (1967), 21-26.

20. __ Applications of sieve methods to the theory of numbers, Cambridge Univ. Press, Cambridge, 1976.

21. J. I. Igusa, Arithmetic variety of moduli for genus two, Ann. of Math. (2) 72 (1960), 612-649.

22. K. Kramer, Arithmetic of elliptic curves upon quadratic extension, Trans. Amer. Math. Soc. 264 (1981), 121-135.

23. K. Kramer and J. Tunnell, Elliptic curves and local \&-factors, Compositio Math. 46 (1982), 307-352.

24. J. C. Lagarias and A. M. Odlyzko, Effective versions of the Chebotarev density theorem, Algebraic Number Fields (A. Fröhlich, ed.), Academic Press, London and New York, 1977, pp. 409-464.

25. E. Liverance, Heights of Heegner points in a family of elliptic curves, Ph.D. Thesis, University of Maryland, 1992.

26. K. Mahler, Zur Approximation algebrischer Zahlen. I (Über den gröszten Primteiler binärer Formen), Math. Ann. 107 (1933), 691-730.

27. __ Zur Approximation algebrischer Zahlen. III (Über die mittlere Anzahl der Darstellungen groszer Zahlen durch binäre Formen), Acta Math. 62 (1933), 91-166.

28. L. Mai, The analytic rank of a family of elliptic curves, Canad. J. Math. 45 (1993), 847-862.

29. J.-F. Mestre, Courbes elliptiques et groupes de classes d'idéaux de certains corps quadratiques, J. Reine Angew. Math. 343 (1983), 23-35.

30. —_ Rang des courbes elliptiques d'invariant donné, C. R. Acad. Sci. Paris Sér. I 314 (1992), 919-922.

31. 171-174.

32. L. J. Mordell, Diophantine equations, Academic Press, London and New York, 1969.

33. S. Nakano, Construction of pure cubic fields with large 2-class groups, Osaka J. Math. 25 (1988), 161-170.

34. A. Néron, Propriétés arithmetiques de certaines familles de courbes algebriques, Proc. Internat. Congr. (Amsterdam, 1954), vol. III, Noordhoff, Groningen, and North-Holland, Amsterdam, 1956, pp. 481-488.

35. U. Schneiders and H. G. Zimmer, The rank of elliptic curves upon quadratic extension, Computational Number Theory (A. Pethö, M. Pohst, H. C. Williams, and H. G. Zimmer, eds.), Walter de Gruyter, Berlin and New York, 1991, pp. 239-260.

36. C. Schoen, Bounds for rational points on twists of constant hyperelliptic curves, J. Reine Angew. Math. 411 (1990), 196-204.

37. R. J. Schoof, Class groups of complex quadratic fields, Math. Comp. 41 (1983), 295-302.

38. J.-P. Serre, Lectures on the Mordell-Weil theorem, Vieweg, Braunschweig/Wiesbaden, 1989.

39. T. Shioda, An infinite family of elliptic curves over $\mathbb{Q}$ with large rank via Néron's method, Invent. Math. 106 (1991), 109-119. 
40. J. H. Silverman, Heights and the specialization map for families of abelian varieties, J. Reine Angew. Math. 342 (1983), 197-211.

41. _ Divisibility of the specialization map for families of elliptic curves, Amer. J. Math. 107 (1985), 555-565.

42. __, The arithmetic of elliptic curves, Graduate Texts in Math., vol. 106, Springer-Verlag, Berlin, New York, and Heidelberg, 1985.

43. C. L. Stewart, On the number of solutions of polynomial congruences and Thue equations, J. Amer. Math. Soc. 4 (1991), 793-835.

44. J. Tate, Algorithm for determining the type of a singular fiber in an elliptic pencil, Modular Functions of One Variable. IV (B. J. Birch and W. Kuyk, eds.), Lecture Notes in Math., vol. 476, Springer-Verlag, New York and Heidelberg, 1975, pp. 33-52.

45. J. T. Tate and I. R. Shafarevich, The rank of elliptic curves, Soviet Math. Dokl. 8 (1967), 917-920.

46. J. Top, Neron's proof of the existence of elliptic curves over $\mathbb{Q}$ with rank at least $11, \mathbf{R}$. U. Utrecht Dept. of Math. preprint series, no. 476, 1987.

47. D. Zagier and G. Kramarz, Numerical investigations related to the L-series of certain elliptic curves, J. Indian Math. Soc. 52 (1987), 51-69.

Department of Pure Mathematics, The University of Waterloo, Waterloo, Ontario, CanAda N2L 3G1

E-mail address: cstewart@watserv1. uwaterloo.ca

VAKgroep Wiskunde, University of Groningen, P.O. Box 800, 9700 AV Groningen, The NETHERLANDS

E-mail address: top@math.rug.nuI 